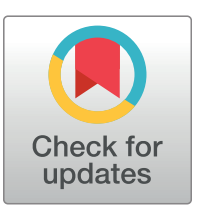

\section{OPENACCESS}

Citation: Chabrolles H, Auclair H, Vegna S, Lahlali T, Pons C, Michelet M, et al. (2020) Hepatitis B virus Core protein nuclear interactome identifies SRSF10 as a host RNA-binding protein restricting HBV RNA production. PLoS Pathog 16(11): e1008593. https://doi.org/10.1371/journal. ppat. 1008593

Editor: Jianming Hu, The Pennsylvania State University College of Medicine, UNITED STATES

Received: April 30, 2020

Accepted: October 4, 2020

Published: November 12, 2020

Copyright: @ 2020 Chabrolles et al. This is an open access article distributed under the terms of the Creative Commons Attribution License, which permits unrestricted use, distribution, and reproduction in any medium, provided the original author and source are credited.

Data Availability Statement: All relevant data are within the manuscript and its Supporting informations files.

Funding: This work was funded by Institut National de la Santé et de la Recherche Médicale (INSERM), the Centre National de la Recherche Scientifique (CNRS), Université Claude Bernard Lyon 1 (UCBL) (AS, DD, FZ, FLC), and the Canadian Institutes of Health Research (BC). It was also supported by grants from Assembly Biosciences (FZ, DD), the

RESEARCH ARTICLE

\title{
Hepatitis B virus Core protein nuclear interactome identifies SRSF10 as a host RNA- binding protein restricting HBV RNA production
}

\begin{abstract}
Hélène Chabrolles $\mathbb{D}^{1}$, Héloïse Auclair ${ }^{1 \odot}$, Serena Vegna ${ }^{1 \odot}$, Thomas Lahlali $\mathbb{D}^{1}$, Caroline Pons ${ }^{1}$, Maud Michelet ${ }^{1}$, Yohann Couté $^{2}$, Lucid Belmudes ${ }^{2}$, Gilliane Chadeuf $\mathbb{B}^{3}$, Yujin Kim $\mathbb{D}^{1}$, Ariel Di Bernardo ${ }^{1}$, Pascal Jalaguier ${ }^{1}$, François-Loïc Cosset $\mathbb{D}^{4}$,

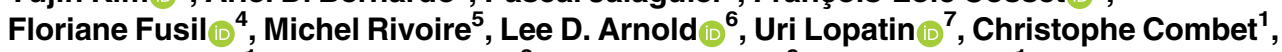
Fabien Zoulim $\oplus^{1}$, David Grierson $\oplus^{8}$, Benoit Chabot ${ }^{9}$, Julie Lucifora', David Durantel $\mathbb{D}^{1 *}$, Anna Salvetti $\mathbb{D}^{1 *}$

1 INSERM, U1052, Cancer Research Center of Lyon (CRCL), Université de Lyon (UCBL1), CNRS UMR5286, Centre Léon Bérard, Lyon, France, 2 Univ. Grenoble Alpes, CEA, INSERM, IRIG, BGE, Grenoble, France, 3 INSERM U1087, Institut du Thorax, Université de Nantes, CNRS UMR6291, Nantes, France, 4 INSERM, U1111, International Center for Infectiology Research (CIRI), Université de Lyon (UCBL1), CNRS, UMR5308, Ecole Normale Supérieure de Lyon, Lyon, France, 5 INSERM U1032, Centre Léon Bérard (CLB), Lyon, France, 6 DiscoverElucidations, LLC, Rancho Santa Fe, California, United States of America, 7 Assembly Biosciences, San Francisco, California, United States of America, 8 Faculty of Pharmaceutical Sciences, University of British Columbia, Vancouver, British Columbia, Canada, 9 Department of Microbiology and Infectious Diseases, Faculty of Medicine and Health Sciences, Université de Sherbrooke, Sherbrooke, Quebec, Canada
\end{abstract}

๑ These authors contributed equally to this work.

* david.durantel@inserm.fr (DD); anna.salvetti@inserm.fr (AS)

\section{Abstract}

Despite the existence of a preventive vaccine, chronic infection with Hepatitis B virus (HBV) affects more than 250 million people and represents a major global cause of hepatocellular carcinoma ( $\mathrm{HCC}$ ) worldwide. Current clinical treatments, in most of cases, do not eliminate viral genome that persists as a DNA episome in the nucleus of hepatocytes and constitutes a stable template for the continuous expression of viral genes. Several studies suggest that, among viral factors, the HBV core protein $(\mathrm{HBc})$, well-known for its structural role in the cytoplasm, could have critical regulatory functions in the nucleus of infected hepatocytes. To elucidate these functions, we performed a proteomic analysis of $\mathrm{HBc}$-interacting host-factors in the nucleus of differentiated HepaRG, a surrogate model of human hepatocytes. The HBc interactome was found to consist primarily of RNA-binding proteins (RBPs), which are involved in various aspects of mRNA metabolism. Among them, we focused our studies on SRSF10, a RBP that was previously shown to regulate alternative splicing (AS) in a phosphorylation-dependent manner and to control stress and DNA damage responses, as well as viral replication. Functional studies combining SRSF10 knockdown and a pharmacological inhibitor of SRSF10 phosphorylation (1C8) showed that SRSF10 behaves as a restriction factor that regulates HBV RNAs levels and that its dephosphorylated form is likely responsible for the anti-viral effect. Surprisingly, neither SRSF10 knock-down nor 1C8 
Agence Nationale de Recherche sur le Sida et les hépatites virales (ANRS; ECTZ51075, to AS), FINOVI (R18051CC to AS), and by the Agence Nationale de la Recherche (ANR; ANR-10-INBS-0801 to $\mathrm{YC})$. The funders had no role in study design, data collection and analysis, decision to publish, or preparation of the manuscript.

Competing interests: I have read the journal's policy and the authors of this manuscript have the following competing interests: Uri Lopatin is an advisor to and shareholder of Assembly Biosciences. Lee Arnold was an employee of Assembly Biosciences. treatment modified the splicing of HBV RNAs but rather modulated the level of nascent HBV RNA. Altogether, our work suggests that in the nucleus of infected cells $\mathrm{HBc}$ interacts with multiple RBPs that regulate viral RNA metabolism. Our identification of SRSF10 as a new anti-HBV restriction factor offers new perspectives for the development of new host-targeted antiviral strategies.

\section{Author summary}

Chronic infection with Hepatitis B virus (HBV) affects more than 250 million of people world-wide and is a major global cause of liver cancer. Current treatments lead to a significant reduction of viremia in patients. However, viral clearance is rarely obtained and the persistence of the HBV genome in the hepatocyte's nucleus generates a stable source of viral RNAs and subsequently proteins which play important roles in immune escape mechanisms and liver disease progression. Therapies aiming at efficiently and durably eliminating viral gene expression are still required. In this study, we identified the nuclear partners of the HBV Core protein $(\mathrm{HBc})$ to understand how this structural protein, responsible for capsid assembly in the cytoplasm, could also regulate viral gene expression. The $\mathrm{HBc}$ interactome was found to consist primarily of RNA-binding proteins (RBPs). One of these RBPs, SRSF10, was demonstrated to restrict HBV RNA levels and a drug, able to alter its phosphorylation, behaved as an antiviral compound capable of reducing viral gene expression. Altogether, this study sheds new light on novel regulatory functions of $\mathrm{HBc}$ and provides information relevant for the development of antiviral strategies aiming at preventing viral gene expression.

\section{Introduction}

Despite the existence of a preventive vaccine, chronic infection with Hepatitis B virus (HBV) remains a major health problem worldwide, as it represents a major global cause of hepatocellular carcinoma (HCC) [1]. Clinically approved treatments, mainly based on nucleoside analogs (NUCs), can reduce HBV viremia under the limit of detection in patients [2]. NUCs, while potent, only affect a relatively late step in the viral life cycle, the conversion of viral pregenomic RNA into viral DNA after encapsidation. They have no known effect elsewhere in the viral life cycle, and as a result viral clearance is rarely obtained and rebound off therapy is common, thus making life-long therapy with NUCs mandatory. The persistence of the viral genome (an episome called covalently-closed-circular dsDNA or cccDNA) in the nucleus of non-dividing hepatocytes constitutes one major obstacle toward a complete eradication of $\mathrm{HBV}$ infection. Indeed, cccDNA not only guarantees viral persistence in the organism but also constitutes a stable source of viral protein expression, including the HBe and HBs antigens (HBeAg and HBsAg), which play important roles in immune escape mechanisms and liver disease progression [3]. Therefore, therapies aiming at efficiently and durably blocking the production of viral antigens are still required [4,5].

HBV is a small enveloped, DNA virus that replicates in hepatocytes. After binding to its receptor, the sodium taurocholate co-transporting polypeptide (NTCP), and uncoating, the viral capsid is transported to the nucleus where the viral genome, constituted by a relaxed circular and partially dsDNA molecule of $3.2 \mathrm{~Kb}$ ( $\mathrm{rcDNA}$ ), is released [6]. Conversion of rcDNA into cccDNA occurs in the nucleoplasm via the intervention of cellular enzyme [7-9]. It results 
in the establishment of a viral episome that constitutes the template for the transcription of five RNAs of 3.5 (precore and pregenomic RNA), 2.4, 2.1 and $0.7 \mathrm{~kb}$ that, respectively, encode the $\mathrm{HBeAg}$, Core protein $(\mathrm{HBc})$, viral polymerase, three surface glycoproteins ( $\mathrm{S}, \mathrm{M}$ and $\mathrm{L}$; all defining the $\mathrm{HBsAg}$ ), and X protein (HBx). Importantly, all these RNAs are unspliced. Several other spliced RNA species are also generated. These spliced forms can be detected in the sera and livers of chronically-infected patients as well as in cells transfected with HBV genomes [10]. They are not required for virus replication but could be involved in HBV-induced pathogenesis and disease progression [11]. Formation of new viral particles initiates in the cytoplasm by packaging of the polymerase-bound pregenomic RNA (pgRNA) into the capsid. Reverse transcription of pgRNA into rcDNA occurs within capsids that are then either enveloped and secreted to form progeny viral particles or re-routed toward the nucleus to replenish the cccDNA pool [6].

$\mathrm{HBc}$ is the sole structural component required for the assembly of the capsid [12]. This protein of 183 amino acids (aa) is composed of a N-terminal domain (NTD, aa 1-140) that is essential for the assembly process, and a C-terminal basic domain (CTD, aa 150-183) that is dispensable for assembly. The CTD domain contains motifs responsible for trafficking of the capsid in and out of the nucleus and displays DNA/RNA binding and chaperone activities $[13,14]$. Studies on $\mathrm{HBC}$ assembly have shown that the protein forms homodimers. Capsid assembly is initiated by the slow assembly of a trimer of dimers to which $\mathrm{HBc}$ dimers rapidly associate to form an icosahedral capsid [12]. Packaging of Pol-pgRNA complex that occurs during capsid assembly is mediated by the CTD of $\mathrm{HBc}$, which also regulates reverse-transcription of pgRNA into rcDNA [15-17].

Converging observations suggest that, besides its structural role in the cytoplasm, $\mathrm{HBc}$ may also exhibit important regulatory activities to control the establishment and persistence of $\mathrm{HBV}$ infection. First, following viral entry, $\mathrm{HBc}$, derived from incoming particles, can enter the nucleus together with rcDNA, where it can form dimers/oligomers and also reassemble into "capsid-like" structures $[18,19]$. Nuclear entry of HBc can occur after a de novo infection, or as a consequence of the re-routing of capsids to the nucleus. Accordingly, nuclear $\mathrm{HBc}$ can easily be detected either in vitro, i.e. in experimentally infected human hepatocytes, or in vivo in the livers of chronically infected patients or model animals [20-23]. Second, earlier studies have shown that $\mathrm{HBc}$ binds to cccDNA, and modifies nucleosomal spacing [24,25]. Association of $\mathrm{HBc}$ to cccDNA was further confirmed in vitro and in vivo and correlated to an active transcriptional state [26-28]. Finally, $\mathrm{HBc}$ was also reported to bind to the promoter region of several cellular genes [29]. Altogether, these data strongly suggest that this structural protein may be important at some nuclear steps of the viral life cycle that remain to be clarified.

In order to gain insight into $\mathrm{HBc}$ nuclear functions, we performed a proteomic analysis of its cellular partners in the nucleus of human hepatocytes. Our results revealed that $\mathrm{HBc}$ mainly interacts with a network RNA-binding proteins (RBPs) that are involved in several post-transcriptional processes and in particular, pre-mRNA splicing. Among these RBPs, we identified SRSF10 as a host factor restricting HBV RNA synthesis/accumulation which opens new perspectives for the development of novel antiviral agents.

\section{Results}

\section{Host RNA-binding proteins are major $\mathrm{HBc}$ interacting factors in the nucleus of differentiated hepatocytes}

To gain insight on $\mathrm{HBc}$ regulatory functions, we sought to identify its nuclear host-partners in human hepatocytes. To this end, we used differentiated HepaRG cells (dHepaRG) expressing $\mathrm{HBc}$, fused at its $\mathrm{N}$-terminus to a streptavidin (ST)-binding peptide (dHepaRG-TR-ST-HBc) 
under the control of a tetracyclin-inducible promoter (Fig 1A). The ST-HBc fusion protein localized in the nucleus of hepatocytes (S1A Fig) and assembled into capsid-like structures as wild type (wt) $\mathrm{HBc}$, confirming that addition of a tag at its N-terminus did not alter these functions (S1B Fig) [30]. ST-HBc/host-factor complexes were purified from nuclear extracts on Strep-Tactin affinity columns (Fig $1 \mathrm{~B}$ and Fig $1 \mathrm{C}$ ). The negative control was provided by dHepaRG-TR cells expressing wt $\mathrm{HBc}$, without any tag and thus unable to bind to the affinity column. In addition, to eliminate cellular partners recovered via DNA/RNA bridging, purification of ST-HBc-complexes was also performed on cell lysates submitted to nucleic acid digestion with Benzonase. Three independent purifications of ST-HBc-associated proteins, done with three different HepaRG differentiation batches, were performed in each condition (+/- Benzonase) and eluted proteins were analyzed by mass spectrometry (MS)-based labelfree quantitative proteomics. This analysis resulted in the identification of 60 and 45 proteins found significantly associated with $\mathrm{HBc}$, with and without Benzonase treatment, respectively (p-value $<0.01$ and fold change $>4$ ) (S1 Table). Importantly, 38 of these factors were common to both conditions, demonstrating the reliability of their identification (Fig 1D).

Gene ontology (GO) annotation of HBc-interacting factors, revealed that approximately $50 \%$ of the factors, identified with or without Benzonase treatment and significantly associated with $\mathrm{HBc}$, were nucleic acid binding proteins and belonged to the RBP family. In the presence of Benzonase, the most abundant protein category (Q-value: $1.8 \times 10^{-29}$ ) identified, corresponded to factors involved in RNA post-transcriptional processes, in particular splicing (Fig $2 \mathrm{~A}$ ). The second most-relevant category (Q-value: $4.4 \times 10^{-14}$ ) corresponded to ribosomal proteins. The similarity of the interactome obtained with and without Benzonase suggests that most of these interactions occurred in the absence of nucleic acids or, alternatively, that they formed high order complexes in which the DNA/RNA was protected from nuclease digestion.

As the major GO category corresponded to RBPs involved in splicing, we next focused on proteins corresponding to this functional group and common to conditions with and without Benzonase (i.e. 11 proteins highlighted in bold in Fig 1D). The interactome of these $11 \mathrm{RBPs}$, hereafter designed as "founder" RBPs, showed that they were highly inter-connected and that several of their first-level interacting partners were also found among $\mathrm{HBc}$-co-purified factors (Fig 2B).

The analysis of the relative abundance of these founder RBPs indicated that SRSF10 was the most abundant RBPs co-purified in HBc-complexes, followed by RBMX, SRSF1, SRSF5 and TRA2B (Fig 3A). Western blot analyses confirmed the presence of SRSF10, RBMX, DDX17, SRSF2 and TRA2B in ST-HBc purified complexes, as well as that of two other non-RBP factors, PARP1 and DNAJB6 (Fig 3B and 3C). In contrast, the presence of SRSF1 could not be confirmed by Western blot (Fig 3B). The reason for this lack of detection is presently unclear but it could be due to a poor sensitivity of the antibodies used.

\section{HBc interacts with multiple SRSF10 isoforms}

SRSF10, the most abundant cellular protein in our $\mathrm{HBc}$ nuclear interactome study, is a member of the SR protein family of splicing factors [31,32]. As all the other members of the SR family, SRSF10 is composed of a N-terminal RNA recognition motif (RRM) and a C-terminal arginine an serine-rich domain (RS) that is responsible for binding to other RBPs and that, in the case of SRSF10 is split in two modules, RS1 and RS2 [33]. Two isoforms of SRSF10 of 37 and $20 \mathrm{KDa}$ have been originally described, the smaller presenting a deletion of the C-terminal RS2 domain, but only the full-length has been extensively studied (S2A Fig). The confirmation of the interaction between $\mathrm{HBc}$ and SRSF10 in an infectious cell culture model was extensively studied by co-immunoprecipitation. Using in vitro HBV-infected dHepaRG or freshly isolated 


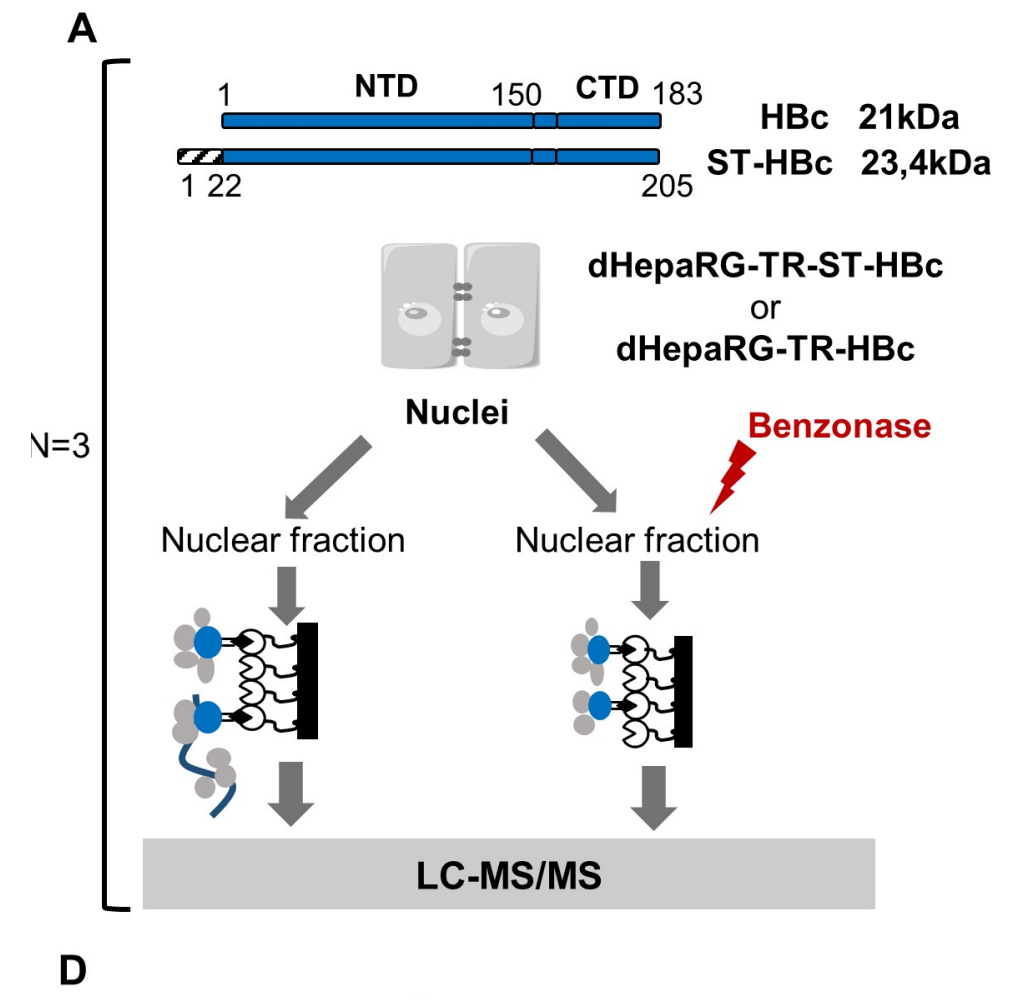

B

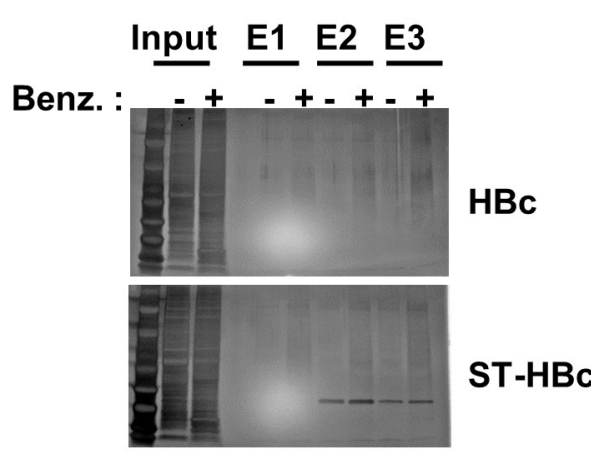

C

Input E1 E2 E3
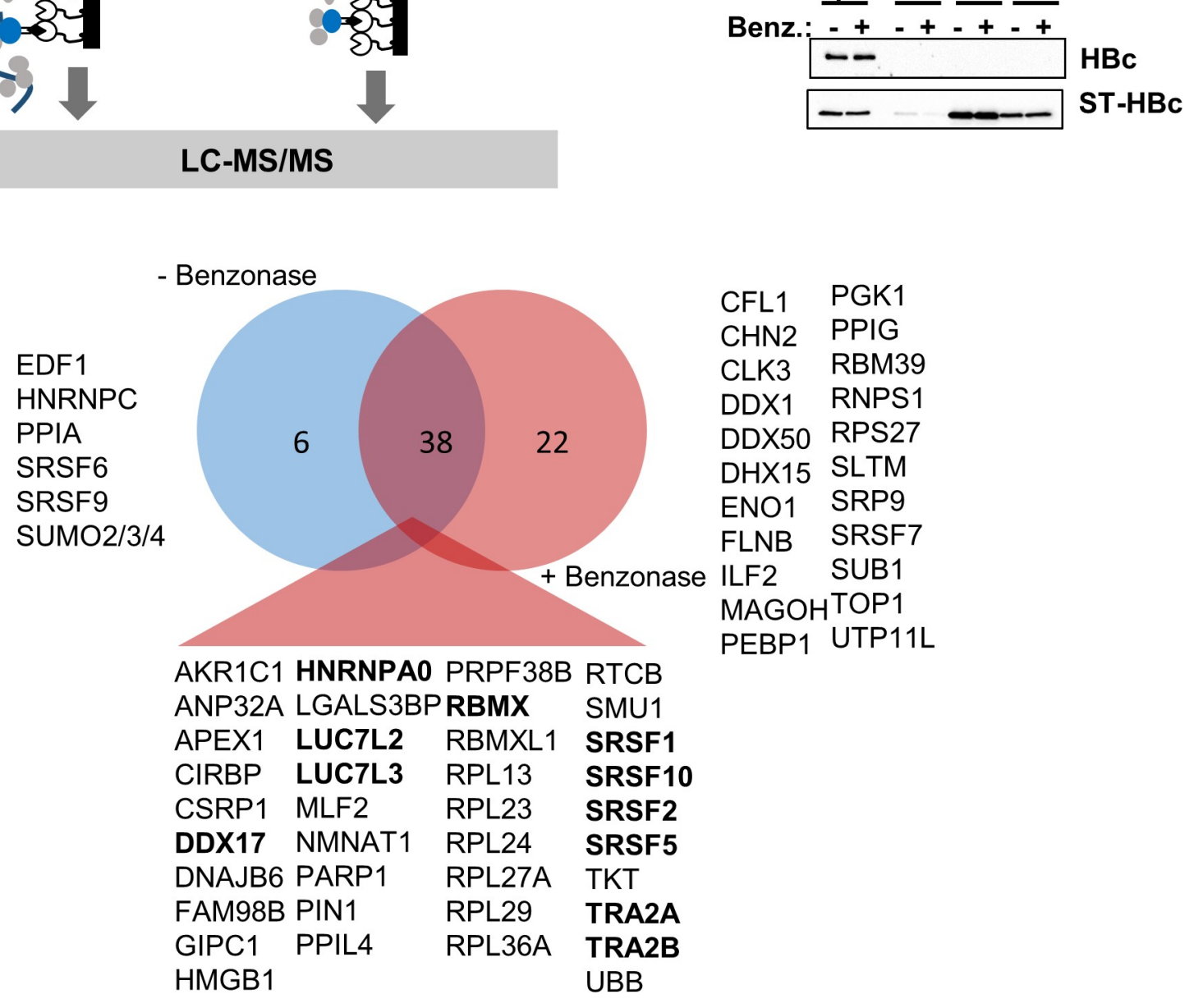

Fig 1. Identification of $\mathrm{HBc}$-interacting proteins in the nucleus of dHepaRG cells. (A) Schematic view of $\mathrm{HBc}$ purification process. Nuclei were purified from differentiated HepaRG-TR cells (dHepaRG-TR) expressing either wt HBc or ST-HBc under the control of a tetracyclin-inducible promoter, lysed and then treated or not with Benzonase. Nuclear extracts were purified on a Streptactin column and protein eluted with desthiobiotin. Input and eluted fractions (E1, E2, and E3) were analyzed by gel electrophoresis followed by silver staining (B) and western blot (C) using an anti-HBc antibody. (D) Venn diagram of proteins significantly associated to $\mathrm{HBc}$ common to conditions with and without Benzonase. Proteins in bold correspond to the 11 "founders" RBP common to both conditions (see text, RBMXL is not highlighted because it was considered as a retrogene of RBMX). 
A

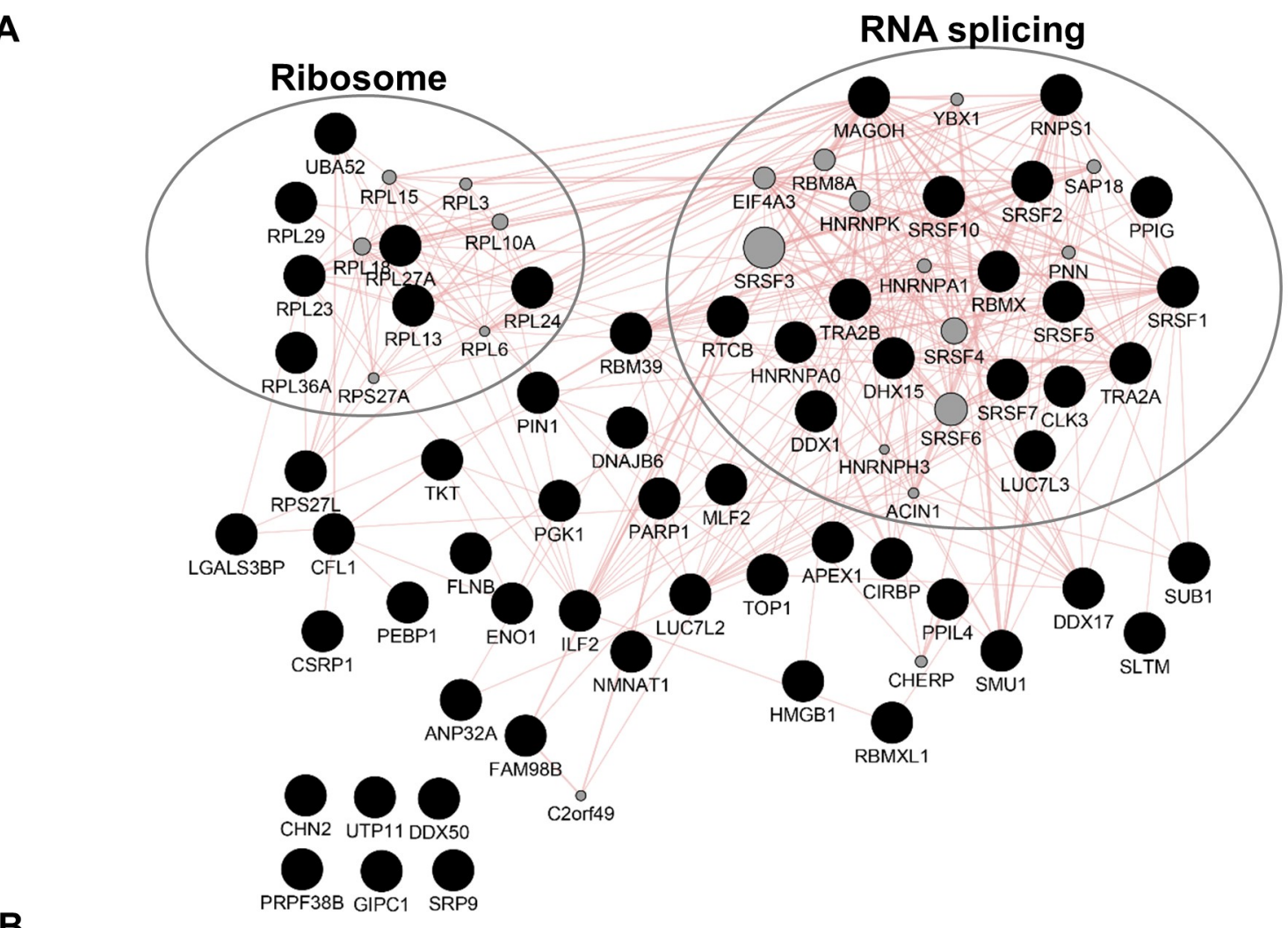

B

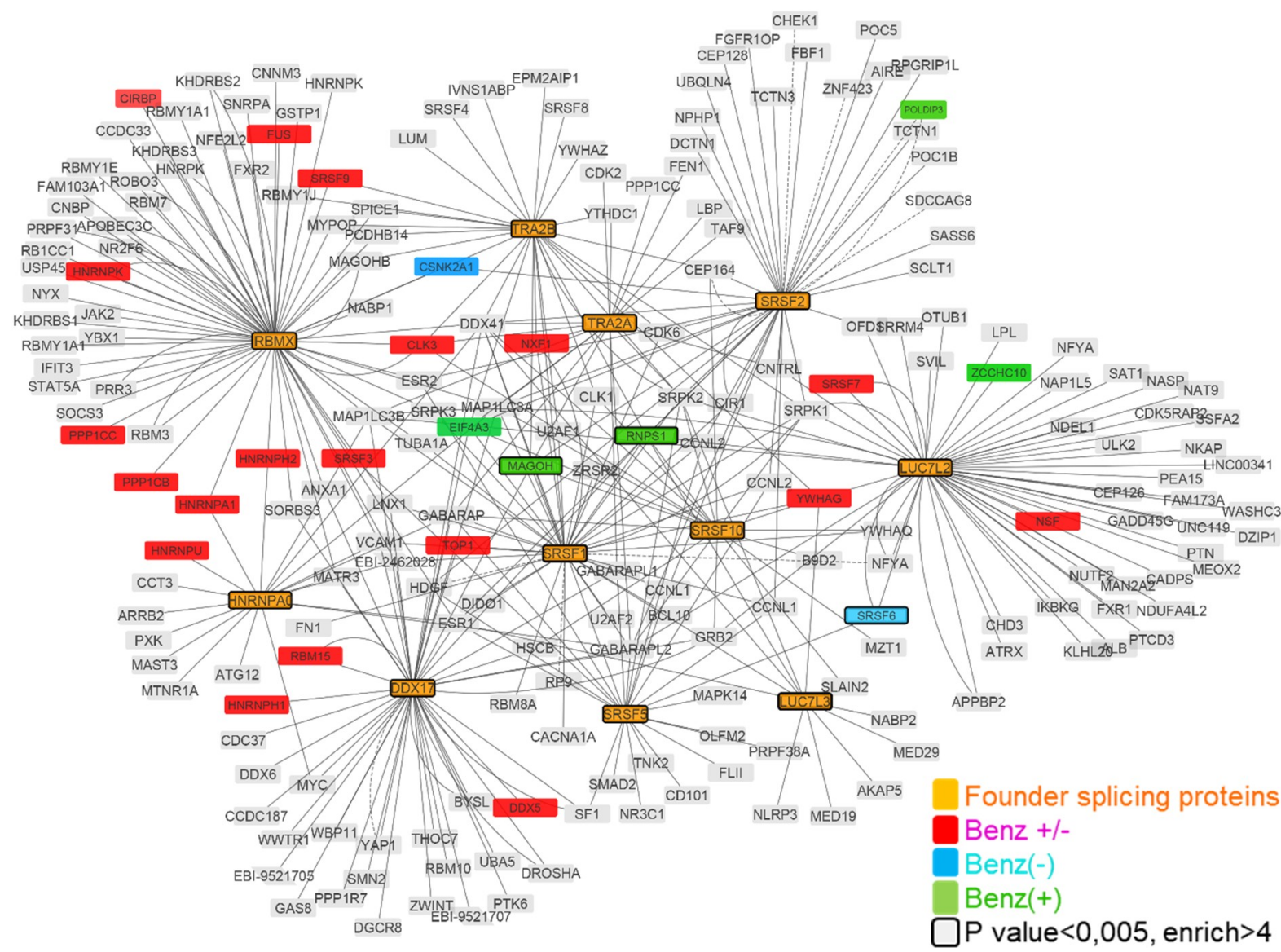

Fig 2. HBc nuclear interactome. (A) Proteins significantly associated to $\mathrm{HBc}$ in the presence of Benzonase were analyzed using the Genemania plugin in Cytoscape (3.7.1). Red lines indicate known physical interactions. Missing nodes are indicated by grey circles. (B) Interaction network of 
proteins involved in mRNA splicing via spliceosome. Significant proteins, common to the Benzonase- $/+$ conditions, over-representing the mRNA splicing via spliceosome biological process ("founder proteins" highlighted as orange nodes) were used to initiate the network by querying IntAct database. Red, blue and green nodes denote protein of the computed network that are found in the proteomic hits of both Benzonase- $/+$ (Benz- or Benz+) conditions. Nodes with a bold border indicate significant proteins ( $p$-value $<0.005$ and fold change $>4$ ) from the proteomics data.

https://doi.org/10.1371/journal.ppat.1008593.g002

A

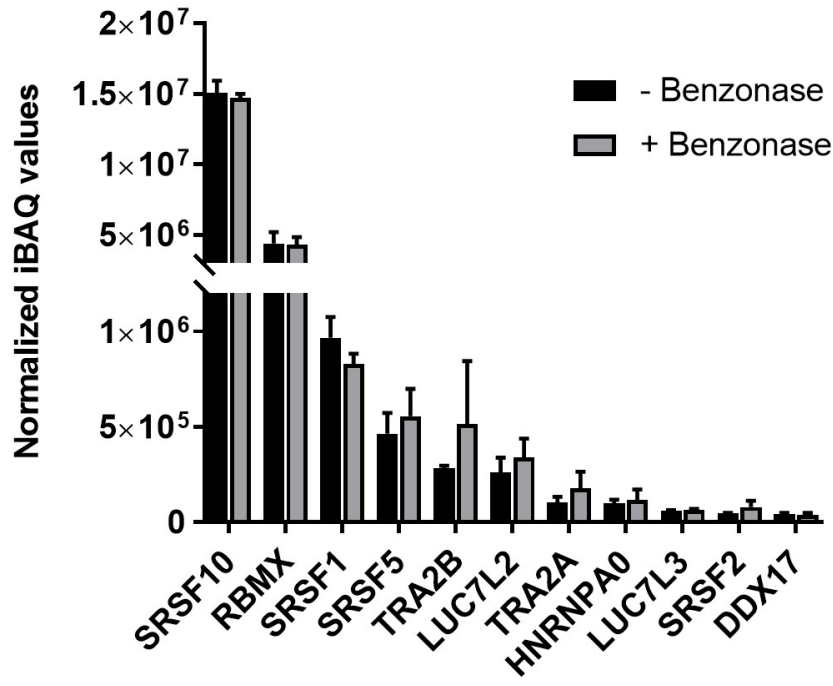

B

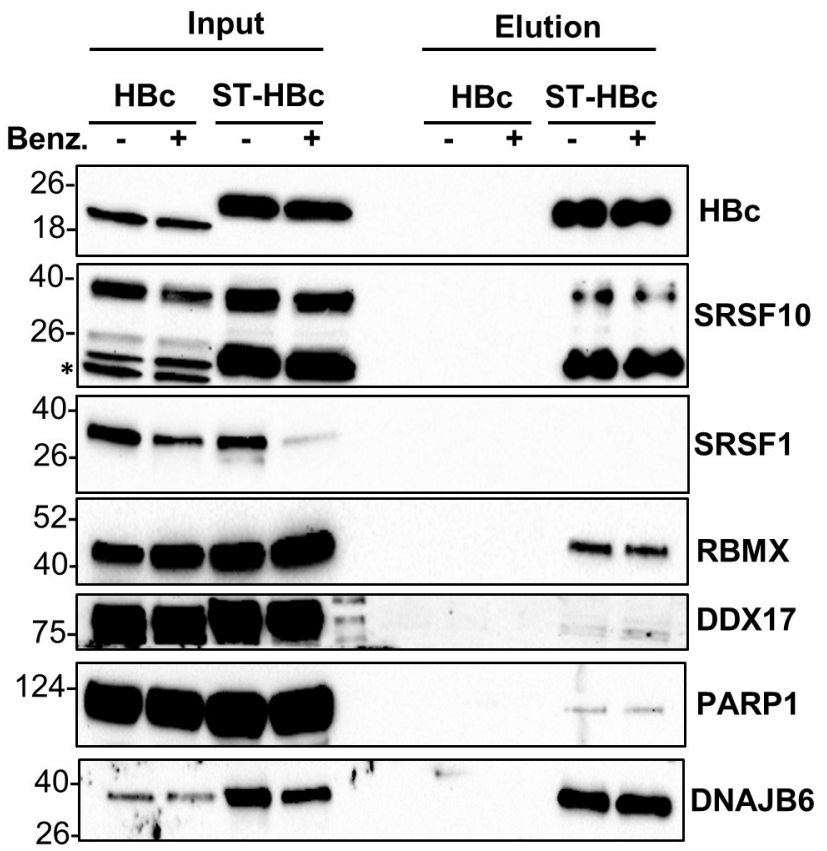

C

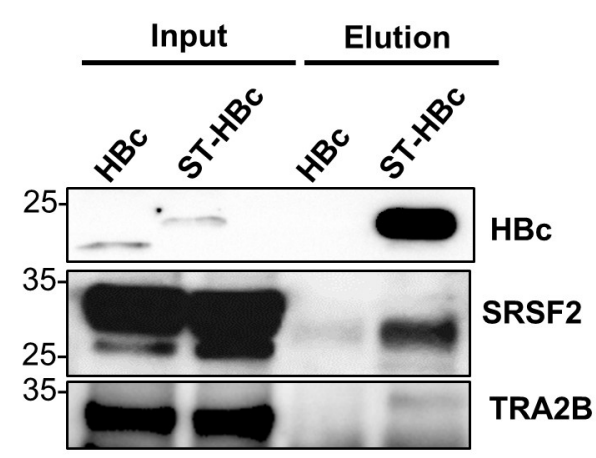

D

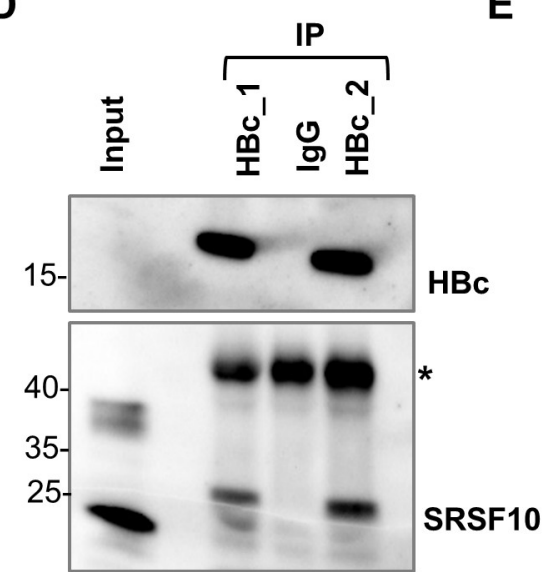

$\mathbf{E}$

\begin{tabular}{|l|l|}
\hline SRSF1 & 7 \\
\hline SRSF10 & 6 \\
\hline UBB & 5 \\
\hline MLF2 $^{*}$ & 10 \\
\hline SRSF9 & 4 \\
\hline SRSF7 & 4 \\
\hline SRSF6 & 4 \\
\hline SRSF2 & 2 \\
\hline
\end{tabular}

Fig 3. Validation analyses. (A) Relative abundances of the 11"founder" RBPs identified in HBc nuclear complexes submitted or not to Benzonase treatment. The relative abundances of $\mathrm{HBc}$ binding partners have been evaluated using the iBAQ metrics [104]. For each replicate, each iBAQ value was normalized by the summed values of the 11 proteins. Error bars represent +/- SD. (B) and (C) Western blot validations in Benzonase treated and streptactin-purified and extracts. Two major isoforms of SRSF10 are visible: the upper at 37KDa and the lower at 20-22 KDa. The band indicated with an asterisk likely corresponds to a band generated by proteolytic cleavage. (D) HBc was immune-precipitated from nuclear extracts purified from liver sections from $\mathrm{HBV}$-infected HuHep mice, using two different anti-HBc antibodies. Eluted proteins were analyzed by western blot using anti $\mathrm{HBc}$ and anti-SRSF10 antibodies. The asterisk indicates the positions of IgG heavy chain. (E) Proteins included in the gel band between 35 and $25 \mathrm{KDa}$ were analyzed by MS. The table indicates the list of proteins recovered with the anti- $\mathrm{HBc}$ antibody that were also previously found after $\mathrm{HBc}$ purification on StrepTactin columns (see Fig 1D). ${ }^{*}$ In the case of MLF, 1 peptide was found in the anti-IgG control IP. The other proteins were found exclusively in the anti-HBc IP. 
primary human hepatocytes $(\mathrm{PHH})$, results were inconclusive, maybe due to the rather low levels of infection in these models. In order to ascertain the interaction between $\mathrm{HBc}$ and SRSF10, co-immunoprecipitation (co-IP) analyses were performed using hepatocytes from mice engrafted with human hepatocytes and infected with HBV (HuHep mice), a model in which the replication level of $\mathrm{HBV}$ is very high. Using these samples, we found that $\mathrm{IP}$ of $\mathrm{HBc}$, performed using two different antibodies led to detection of a band reacting with the anti-SRSF10 antibody but migrating at a size between 25 and $35 \mathrm{KDa}$ that did not correspond to the size of the two major SRSF10 isoforms of 37 and $20 \mathrm{KDa}$ detected in the input fraction (Fig 3D). Because this size was unexpected, proteins present in the gel band included between 35 and $25 \mathrm{KDa}$ were analyzed by MS. This analysis confirmed the presence of SRSF10 that was identified by 6 different peptides, covering the first 100 aa, found exclusively in the anti-HBc sample (Fig 3E). In addition to SRSF10, seven other proteins, among which five SR proteins, previously identified in the ST-HBc eluted fraction (Fig 1D) were also detected. Importantly, coIP performed using dHepaRG-HBc or ST-HBc nuclear extracts similarly detected this new SRSF10 band in addition to the other conventional SRSF10 isoforms (S2B Fig). At least 9 different SRSF10 isoforms, all containing the RRM and RS1 domains, are predicted to be produced from alternatively spliced transcripts (https://www.ncbi.nlm.nih.gov/gene/10772). This new under-represented isoform of approximately 30-32 KDa may correspond to a variant of 217 aa (S2C Fig) that contains the RRM and RS1 domains, as well as a shorter RS2 domain (NP_001300937.2).

Altogether, these results indicated that the interaction between HBc and SRSF10 as well as with other, previously identified SR proteins was maintained in HBV-infected PHH. It also indicated that $\mathrm{HBc}$ can potentially associate with different SRSF10 isoforms further suggesting the potential importance of this interaction for the viral life cycle.

\section{SRSF10 modulates HBV RNA levels}

We next investigated the effect of a SRSF10 knock-down (KD) on HBV infection. Importantly, the siRNAs used to KD SRSF10 localized to the RRM and RS1 coding sequence and thus potentially targeted all the SRSF10 isoforms, including those not visible by Western blot, since they all share these domains (S2A Fig). Optimization of the siRNA transfection protocol led to a significant level of protein KD in PHH without affecting NTCP levels, strongly suggesting that $\mathrm{HBV}$ internalization was not affected (Fig 4A-4C). In PHH, KD of SRSF10 resulted in a significant increased accumulation of total HBV RNAs and pgRNA without affecting cccDNA level (Fig 4D). Similar results were observed in dHepaRG cells with the exception that, in this cell model, a modest but significant increase in cccDNA level, was observed (S3A-S3D Fig). The effect of SRSF10 KD on HBV RNAs was also confirmed by Northern blot confirming the increase of the three detectable HBV RNAs molecules (S4 Fig). In sharp contrast, KD of RBMX resulted in opposite effects on HBV replication, with a decrease of all viral parameters, including cccDNA (Fig 4E and 4F, S3 Fig). These results indicated that RBPs found associated with $\mathrm{HBc}$ play distinct roles in the HBV life cycle. To determine whether SRSF10 KD had similar effect on an already established HBV infection, siRNA-mediated KD was also performed 7 days after the onset of infection, when replication has reached a plateau [34,35]. In dHepaRG cells, a reproducible increase of HBV RNA could be observed following SRSF10 KD even if at a lower level as compared to cells in which the KD was performed before infection (S5 Fig). As previously observed in cells transfected before infection, SRSF10 KD also increased cccDNA level suggesting that, in this cell type, SRSF10 may modulate cccDNA recycling and /or stability in addition to its effect on HBV RNAs.

Finally, to investigate if the SRSF10 effect on HBV RNAs was dependent on $\mathrm{HBc}$, we used $A A V$ vectors to deliver into hepatocytes either a wt or mutated $\mathrm{HBV}$ genome unable to 
A

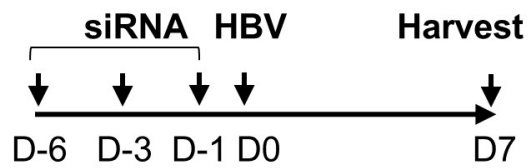

B

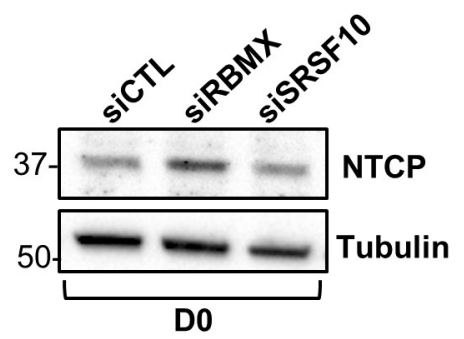

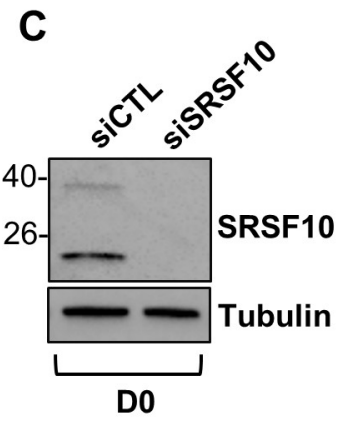

D
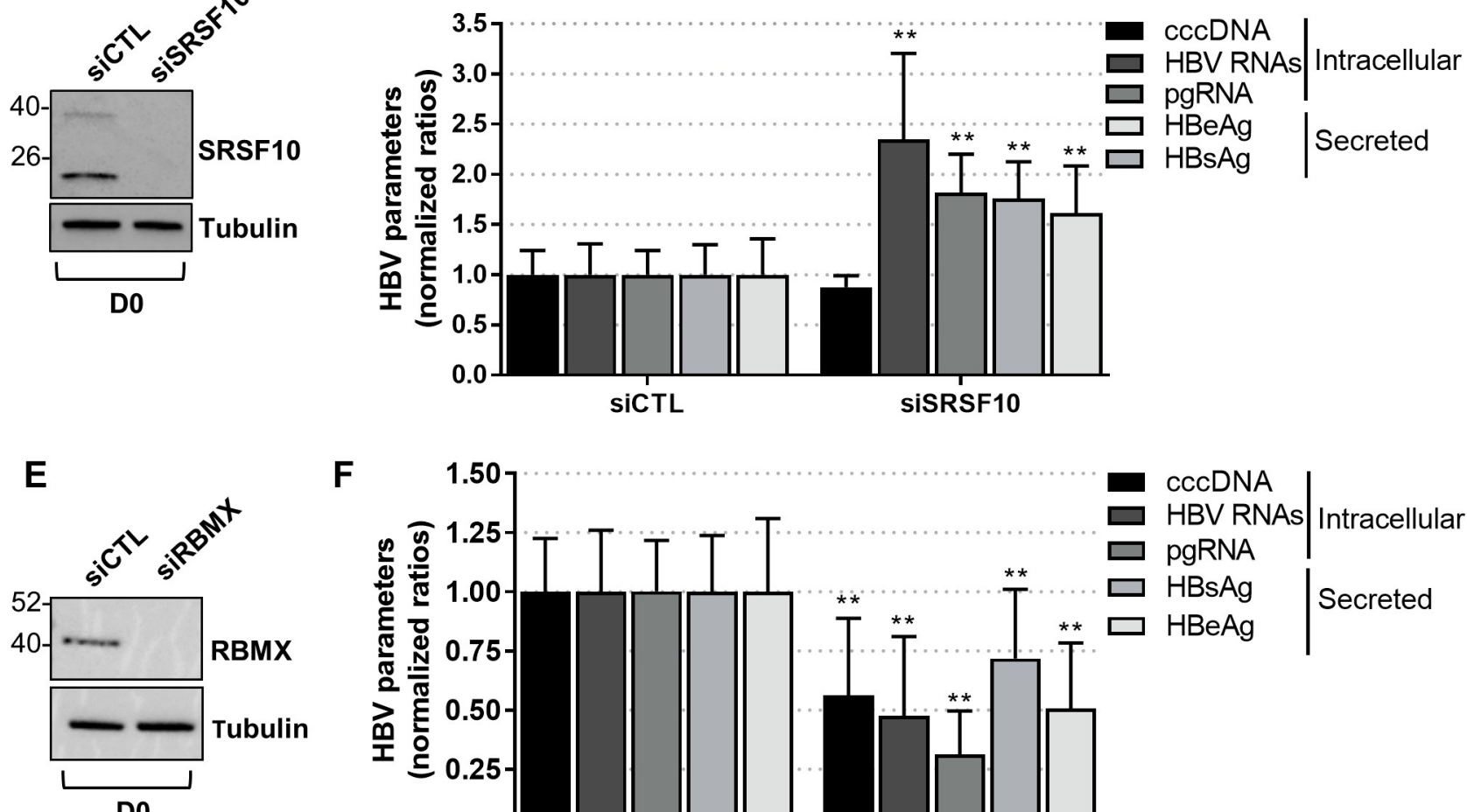

$\mathbf{F}$

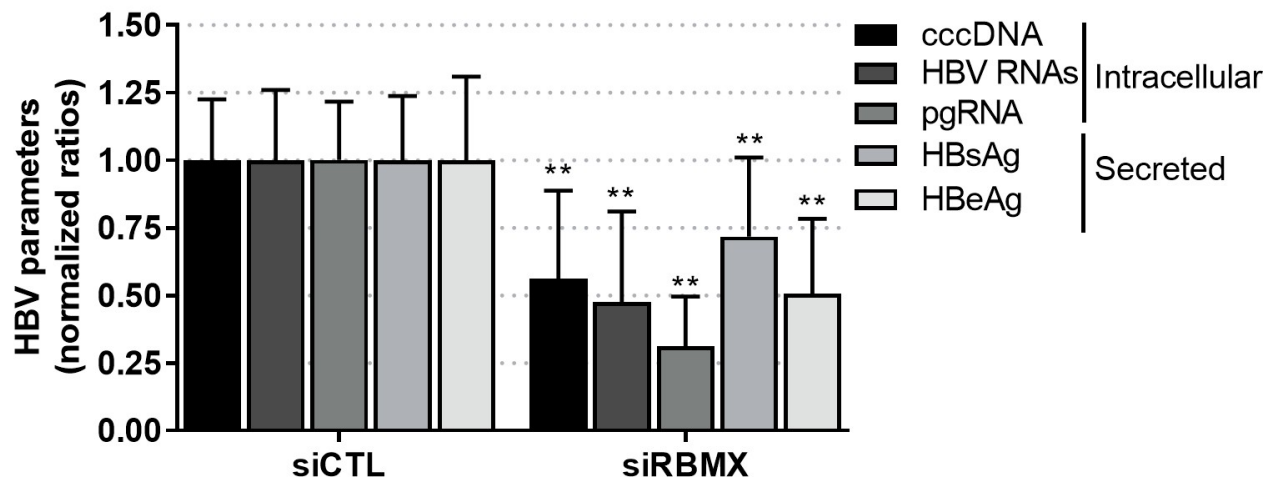

Fig 4. Effect of SRSF10 or RBMX KD on HBV replication in PHH. (A) Outline of the experimental protocol: cells were transfected with siRNA targeting SRSF10 or RBMX or control siRNA (siCTL) and then infected with HBV (MOI of 100 vge/cell). Cells and supernatants were harvested 7 days (D) post-infection (pi) and analyzed to measure intracellular and secreted HBV parameters. (B), (C) and (E) Western bot analysis of NTCP, SRSF10 and RBMX protein levels at D0 of the protocol, respectively. (D) and (F) HBV intracellular and extracellular parameters were measured at D7 pi. Results are expressed as the mean normalized ratio +/- SD, between siSRSF10 or siRBMX and siCTL transfected cells, of 3 independent experiments, each performed in triplicate, with PHH from different donors.

https://doi.org/10.1371/journal.ppat.1008593.g004

produce $\mathrm{HBc}$ and compared their replication level in the presence in the absence of SRSF10 (S6A Fig). In particular two mutated AAV-HBV genomes were used: one with a point mutation of the HBc ATG (AAVHBVnoHBc), and the other with a 406 bp deletion of the HBc coding sequence included between the HBc ATG and the beginning of the polymerase ORF (AAVHBV $\triangle H B c$ ). Transduction of dHepaRG cells with these three vectors led to the establishment of HBV infection as detected by the quantification of HBV RNAs and secreted antigens (S6B-S6D Fig). As expected the AAVHBVnoHBc vector produced both $\mathrm{HBs}$ and $\mathrm{HBe} A g$ whereas only HBsAg was detected using AAVHBV $\triangle \mathrm{HBc}$-transduced cells. KD of SRSF10 increased all viral parameters in AAVHBVwt-transduced cells. Interestingly, the increase in HBV RNAs and secreted antigens measured following KD of SRSF10 was significantly reduced 
in the absence of $\mathrm{HBc}$ suggesting that the SRSF10 anti-viral effect may be partially dependent on $\mathrm{HBc}$.

Altogether these results indicate that SRSF10 behaves as a restriction factor that mainly modulates HBV RNA levels.

\section{A small molecule inhibitor of SRSF10 phosphorylation strongly impairs HBV replication and antigen secretion}

SRSF10 activity was previously shown to be tightly controlled by phosphorylation, which regulates its interaction with other RBPs and splicing activities [36-39]. De-phosphorylation of SRSF10 occurs in response to heat shocks, DNA damage or during mitosis. More recently, compound 1C8 (Fig 5A), was shown to prevent SRSF10 phosphorylation, in particular at serine 133, in the absence of any other detectable effect on other SR proteins, and to inhibit HIV1 replication with a combined effect on HIV-1 transcription and splice site selection likely producing an imbalance in viral protein required for replication [40,41].

Using a two-dimensional gel electrophoresis, we confirmed that $1 \mathrm{C} 8$ could induce the dephosphorylation of SRSF10 in differentiated human hepatocytes (S7 Fig). To explore the effect of $1 \mathrm{C} 8$ on HBV replication we first assessed its effects when added on HBV-infected dHepaRG cells (Fig 5B). In this setting, treatment with $1 \mathrm{C} 8$ resulted in a decrease of viral RNAs and all downstream secreted parameters (Fig 5C). Interestingly, this phenotype was different from that observed with other antiviral compounds such as a NUC (Tenofovir) that uniquely inhibited HBV DNA synthesis, or a Core allosteric modulator (CAM) that additionally inhibited $\mathrm{HBe}$ secretion [42]. This effect, although weaker, was maintained in HBVinfected PHH (Fig 5D), a more relevant/physiologic model to assess the activity of compounds targeting host functions. Dose response analyses in $\mathrm{dHepaRG}$ indicated an effective concentration 50\% (EC50) of approximately 10 and $5 \mu \mathrm{M}$ for HBV RNAs/secreted DNA and HBsAg/ $\mathrm{HBeAg}$, respectively, in the absence of detectable cell cytotoxicity (S8 Fig). In subsequent analyses we sought to determine if $1 \mathrm{C} 8$ was equally active on other HBV genotypes than D that was used in all our previous experiments. In dHepaRG cells we found that $1 \mathrm{C} 8$ could inhibit the replication of $\mathrm{HBV}$ genotype $\mathrm{C}$, with a significant decrease of viral RNAs and all secreted parameters (S9A Fig). A preliminary analysis with five other HBV genotypes also indicated that $1 \mathrm{C} 8$ could significantly reduce $\mathrm{HBS}$ and $\mathrm{HBeAg}$ secretion in particular for genotypes $\mathrm{G}$ and $\mathrm{H}$, suggesting that its effect may be pan-genotypic (S9B Fig).

These results indicate that $1 \mathrm{C} 8$ can inhibit $\mathrm{HBV}$ replication by reducing HBV RNA levels. The inhibitory effect of 1C8 on HBV RNAs, opposite to that observed following SRSF10 KD, suggests that the de-phosphorylated form of SRSF10, that is depleted following siRNA transfection and, in contrast, induced after 1C8 treatment, is responsible for the observed antiviral activities of this cellular RBP.

\section{$1 C 8$ antiviral effect is partially dependent on SRSF10, promoting a reduction in HBV RNAs but not their splicing}

To verify if the effect of 1C8 on HBV RNA accumulation was indeed related to SRSF10, experiments combining SRSF10 KD and 1C8 treatment were conducted (Fig 6A). Based on the model proposed, the inhibitory effect of $1 \mathrm{C} 8$ on viral RNA production, should be prevented by depleting SRSF10. As previously observed, each treatment alone, 1C8 or siSRSF10, resulted in opposite effects on HBV RNA levels. Remarkably, in cells receiving both treatments, depletion of SRSF 10 could partially rescue the inhibitory effect of 1C8 to a level similar to that observed in control cells without, however, reaching that measured in siSRSF10-transfected cells (Fig 6B and $6 \mathrm{C}$ ). This result indicates that the antiviral effect of $1 \mathrm{C} 8$ is dependent on SRSF10. The lack 
A<smiles>Cn1ccc(=O)c(C(=O)Nc2nsc3ccc([N+](=O)[O-])cc23)c1</smiles>

B

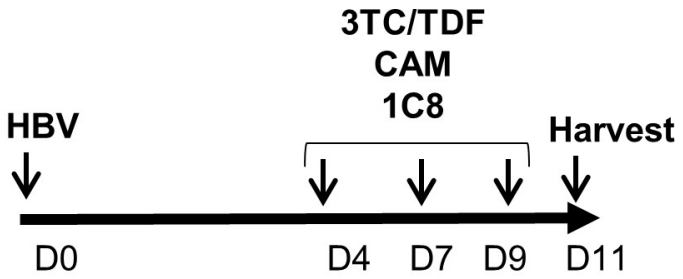

C
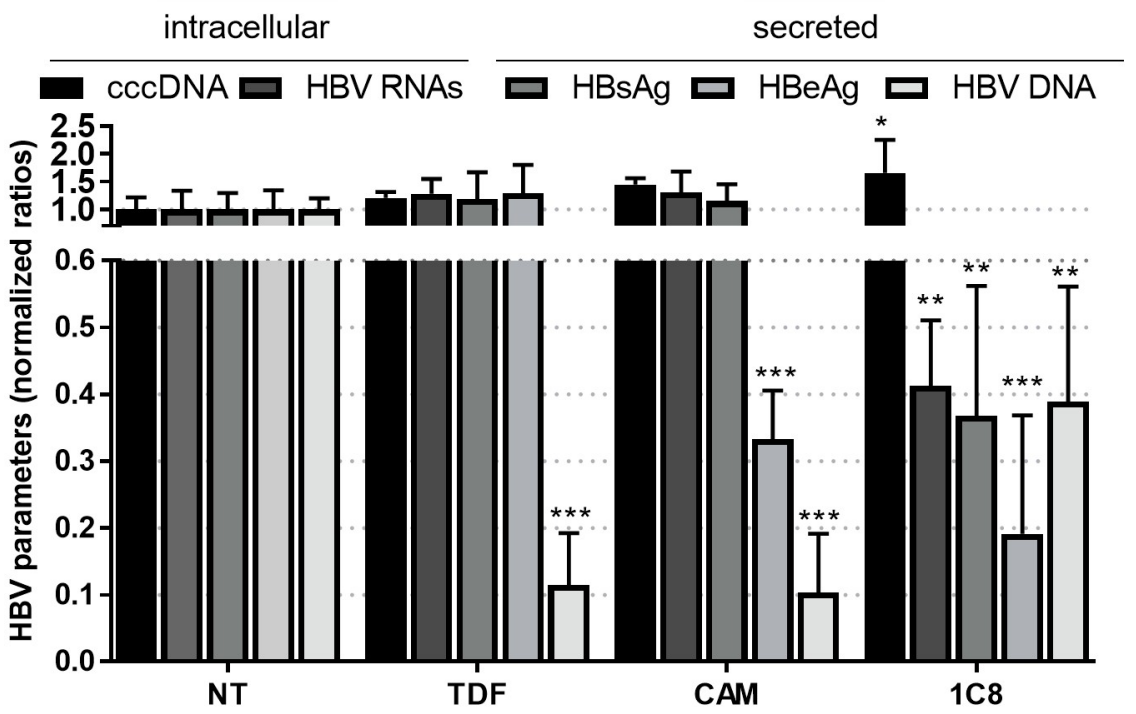

D

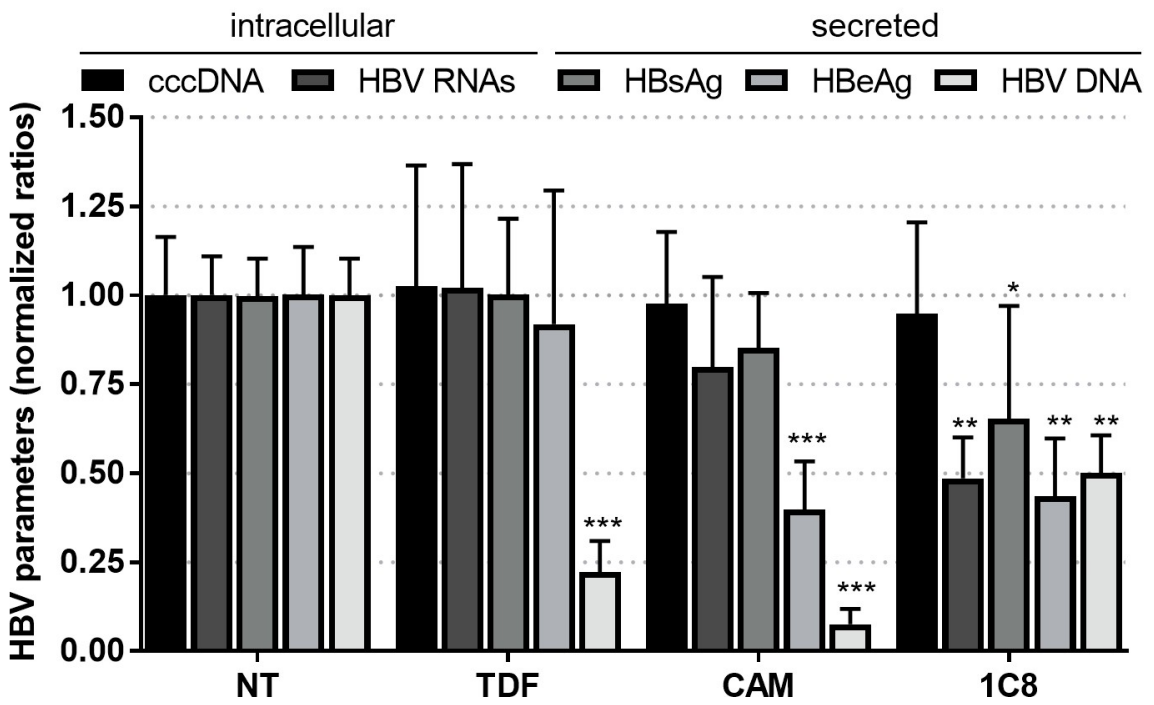

Fig 5. Effect of $1 \mathrm{C} 8$ on an established HBV infection. (A) Molecular structure of 1C8. (B) Outline of the experimental protocol: HBV-infected dHepaRG cells (C) or PHH (D) were treated three times with Tenofovir (TDF at $10 \mu \mathrm{M})$, a Core allosteric modulator $(\mathrm{CAM}$ at $10 \mu \mathrm{M})$ or $1 \mathrm{C} 8(10 \mu \mathrm{M})$ starting at D4pi. Intracellular and secreted HBV parameters were quantified 2 days after the last treatment. Results are expressed as the mean normalized ratio +/- SD between non-treated and treated cells of 3 independent experiments, each performed in triplicate.

https://doi.org/10.1371/journal.ppat.1008593.g005

of a complete rescue, in cells treated with $1 \mathrm{C} 8$ and depleted of SRSF10, could be explained by the persistence of a low level of dephosphorylated SRSF10. Alternatively, it is possible that 1C8 additionally targets other cellular and/viral factors that are involved in the anti-viral effect. 
A

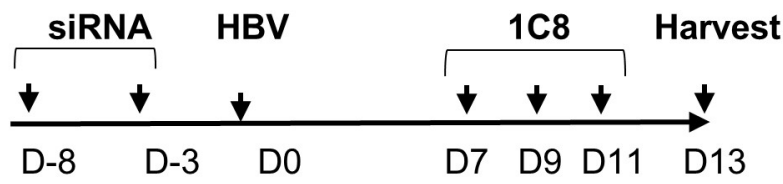

B

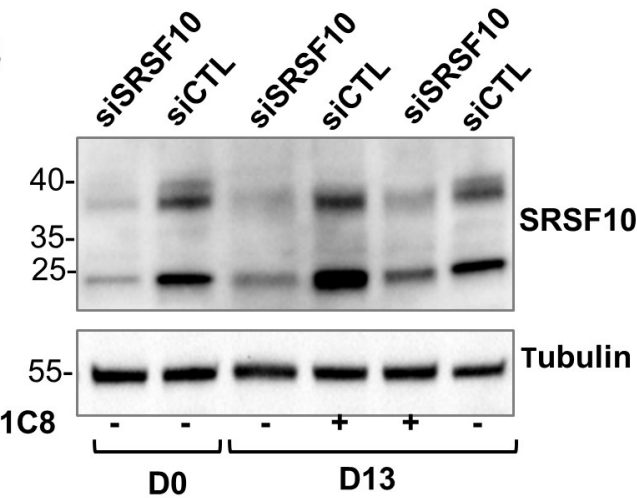

C

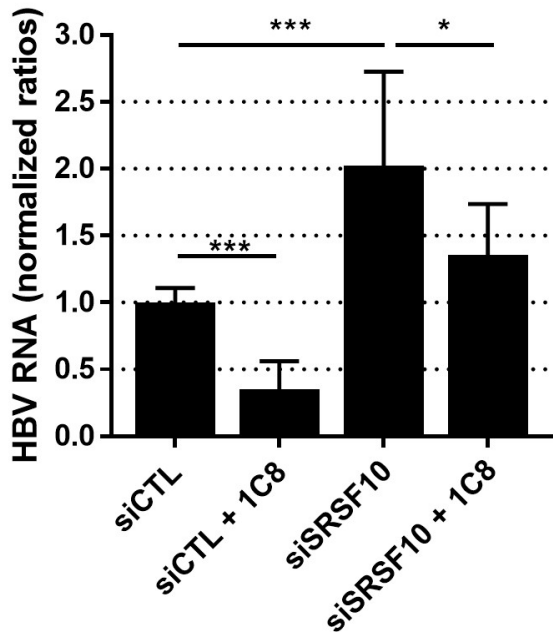

Fig 6. Combined effect of SRSF10 KD and $1 \mathrm{C} 8$ treatment on HBV-infected dHepaRG cells. (A) Outline of the experimental procedure: dHepaRG cells were transfected once or twice with siRNA targeting SRSF10, then infected with HBV (MOI of 250 vge/cell), and 7 days later treated three times with 1C8 $(10 \mu \mathrm{M})$. (B) Western blot validation showing SRSF10 depletion. (C) Quantification of intracellular HBV RNAs. Results are expressed as the mean normalized ratio +/- SD between treated and/or siSRSF10 transfected cells and siCTLtransfected cells of 3 independent experiments, each performed in triplicate.

https://doi.org/10.1371/journal.ppat.1008593.g006

All HBV RNAs required and sufficient for a productive replication (i.e. production of virion and viral proteins) are unspliced. Nonetheless, several spliced HBV mRNA have been documented in experimental models and more importantly in patient samples, indicating that, if an active mechanism of escape from splicing exists, it must be partial and/or ineffective at a certain stage during chronic infection [10]. Among the numerous HBV spliced RNAs, two major spliced forms result in the production of new viral proteins, some being potentially involved in viral pathogenesis, and particles containing shorter viral genomes $[10,43,44]$. In our previous assays, the primers used to quantify HBV RNAs (total and pgRNA) localized to an unspliced region of the HBV genome. Therefore, it was possible that the variations in HBV RNA levels observed after SRSF10 KD or 1C8 treatment could be due to a specific modulation in some spliced variants or to a differential effect on spliced versus unspliced forms. To explore this possibility RNA extracted from siRNA transfected hepatocytes were analyzed by RT-qPCR using primers able to specifically detect each spliced and unspliced RNA (S2 Table). Unexpectedly, the relative quantification of each RNA variant in SRSF10-depleted versus control cells resulted in a global increase of all HBV RNA variants, in particular in $\mathrm{PHH}$, including all detected spliced forms without inducing a preferential modulation of a spliced versus unspliced variants (S10A and S10B Fig). Similarly, treatment of HBV-infected dHepaRG cells with 1C8 post-infection resulted in a strong reduction of all viral RNA whether spliced or unspliced (S10C Fig). These results indicated that the respective proviral or antiviral effect of SRSF10 KD or 1C8 was not associated to a variation in the level of spliced versus unspliced HBV RNAs. They also suggested that both treatments acted on HBV RNAs synthesis and/or stability. To verify this point, total and nascent HBV RNAs were quantified following SRSF10 KD or 1C8 treatment. The quantification of nascent HBV RNAs was performed by labeling newly transcribed RNAs with 
A

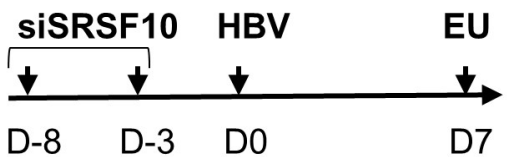

C

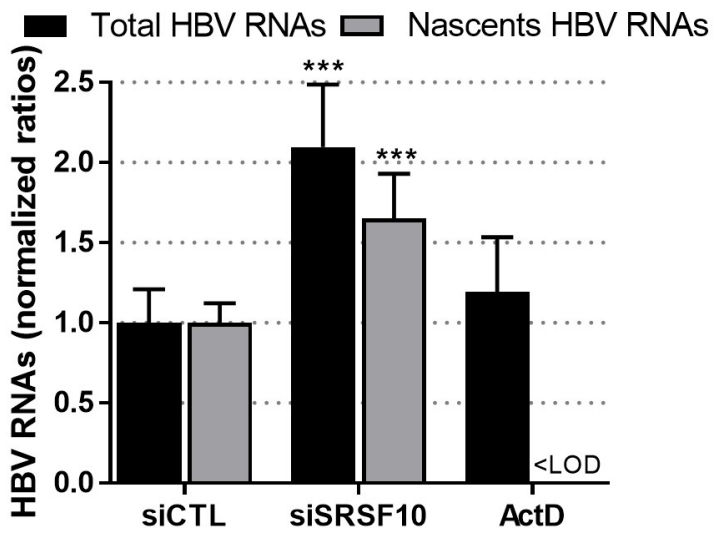

B

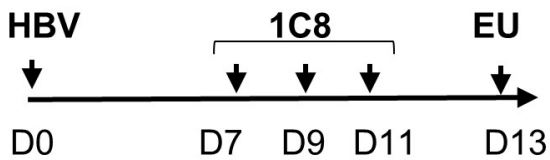

D

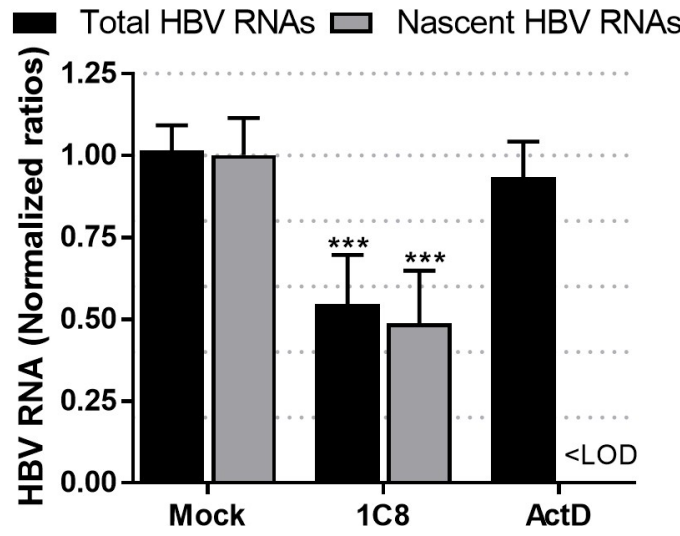

Fig 7. Analysis of nascent HBV RNAs following SRSF10 KD or 1 C8 treatment. (A) dHepaRG cells were transfected with siRNA against SRSF10 and then infected with HBV (MOI of 250 vge/cell). Edu labelling was performed at D7pi for 2 hours. (B) dHepaRG cells were infected with HBV and then treated three times with $1 \mathrm{C} 8(40 \mu \mathrm{M})$ at D7, D9 and D11pi. EU incorporation was performed at D13pi for 2 hours. (C) and (D) Run-on analyses. Intracellular RNA was extracted from transfected/treated cell cells and either directly quantified using HBV primers (Total HBV RNAs) or purified using the Click-iT Nascent RNA Capture kit to quantify newly synthetized RNAs (nascent HBV RNAs). Control was provided by treating cells with Actinomycin D (ActD at $10 \mathrm{mg} / \mathrm{ml}$ ) added to cells 20 min before labeling (see Methods). <LOD: under the limit of detection.

https://doi.org/10.1371/journal.ppat.1008593.g007

ethynyl uridine (EU) for 2 hours before capture (Fig 7A and 7B). As expected, depletion of SRSF10 in dHepaRG cells prior to HBV infection increased total HBV RNAs. Actinomycin $\mathrm{D}$ (ActD), a global transcription inhibitor, strongly reduced the level of nascent RNA. In contrast, in cells transfected with siSRSF10, newly transcribed HBV RNAs were increased at a level similar to that observed for total RNAs (Fig 7C). The same analysis performed on 1C8-treated cells indicated that the compound equally reduced total and nascent HBV RNAs (Fig 7D). Altogether, these analyses indicate that SRSF10 and 1C8 did not modify the splicing level of HBV RNAs but, rather, that both treatments exert their effect by modifying the transcription and/or the stability of nascent viral RNAs.

\section{Discussion}

Our strategy to expand the knowledge on $\mathrm{HBc}$ nuclear functions was based on the identification of its host protein partners in the nucleus of dHepaRG cells, when expressed alone in the absence of other viral constituents. HepaRG cells represented one of the best alternative models to freshly isolated human hepatocytes, and could be easily engineered as opposed to $\mathrm{PHH}$ [45]. This analysis indicated that in the nucleus $\mathrm{HBc}$ interacts with RBPs that are mainly involved in all steps of mRNA metabolism (Fig 2). Importantly, some of these factors were equally identified as $\mathrm{HBc}$-interacting partners in hepatocytes from $\mathrm{HBV}$-infected HuHep mice (Fig 3E). Therefore, even if most of these interactions need to be further validated in other relevant models, the results of both analyses suggest that these cellular RBPs play a role during viral replication. 
Most of these RBPs localize in well-defined nuclear bodies, in particular speckles and paraspeckles $[46,47]$. Recent studies have highlighted that many active genes are located in proximity to nuclear speckles and that this association results in an increase of nascent transcript levels $[48,49]$. The association of $\mathrm{HBc}$ with cellular RBPs strongly suggests that it may intervene in the metabolism of viral RNAs by interacting with a highly interconnected network of proteins that can act at several transcriptional and post-transcriptional steps [50]. Accordingly, $\mathrm{HBc}$ has several features similar to those found in many cellular RBPs with, notably, a positively-charged CTD composed by a long stretch of arginines separated by seven serine residues resembling the arginine/serine-rich domain (RS domain) of several RBPs in particular of SR proteins $[17,51,52]$. When expressed in bacteria, HBc, thanks to its CTD, displays a strong RNA-binding activity [53]. Interestingly, several studies have shown that $\mathrm{HBc}$ also has a strong affinity for DNA, and can associate with cccDNA both in vitro and in vivo [24-27]. Although no evidence yet support the binding of $\mathrm{HBc}$ to viral and/or cellular RNA in the nucleus of HBV-infected hepatocytes, $\mathrm{HBc}$, like some RBPs, may possess the dual ability to bind to DNA and RNA [54]. This activity might be tightly modulated by the phosphorylation level of its $\mathrm{CTD}$, as previously demonstrated during pgRNA packaging [17]. Alternatively, the association of $\mathrm{HBc}$ with cccDNA and/or RNA may be indirectly mediated via RNA molecules and/or its interaction with cellular RBPs. Interestingly, some RBPs found associated to $\mathrm{HBc}$, such as RBMX (Fig 1) were also reported to bind to DNA, in particular during DNA repair [55,56].

Among the RBPs interacting with $\mathrm{HBc}$, we focused on SRSF10, a member of the SR proteins family that was the most highly abundant in $\mathrm{HBc}$-containing complexes. Initial studies identified SRSF10 as a splicing repressor when de-phosphorylated in response to heat shock [39,57]. Further analyses showed that SRSF10 is a regulator of AS, depending on its phosphorylation level that also determines its interaction with diverse RBPs, in particular TRA2A, TRA2B, hnRNPK, F, and H $[36,39,58]$. Importantly, SRSF10 influences the AS of several cellular transcripts involved in pathways of stress response, DNA damage response, apoptosis, and carcinogenesis [58-60]. In accordance to its role in the stress response, SRSF10 was described as a component of paraspeckles, nuclear stress bodies and cytoplasmic stress granules [61-63]. SRSF10 also plays a role in the control of viral RNA, in particular of HIV-1 [41,64]. As all the other members of the SR family, SRSF10 is composed of a N-terminal RNA recognition motif (RRM) and a C-terminal RS domain that is responsible for binding to other RBPs [33]. Two isoforms of SRSF 10 of 37 and $20 \mathrm{KDa}$ have been originally described corresponding to those detected by available antibodies, the smaller presenting a deletion of the C-terminal domain (S2A Fig). In co-IP analyses performed on liver samples from HBV-infected HUHep mice, we found that $\mathrm{HBc}$ mainly interacted with a new SRSF10 isoform with an apparent molecular weight of $32 \mathrm{KDa}$ that was equally visible when co-IP was performed on nuclear extracts from HBc-expressing dHepaRG cells (S2B Fig). The identity of this new isoform is presently unclear but it may correspond to one of the 9 protein variants that can be generated by AS of the SRSF10 pre-mRNA. Why this isoform was detected only after IP and not following purification on StrepTactin columns (Fig 3B) is presently unclear. One hypothesis is that $\mathrm{HBc}$ may differentially interact with various SRSF10 isoforms according to its assembly state (dimer, oligomer or capsid-like structures), and that the ratio of these $\mathrm{HBc}$ forms may vary depending on the purification procedure.

To investigate the role of SRSF10 during HBV replication we analyzed the consequences of its KD in differentiated hepatocytes. Depletion of SRSF10 induced a reproducible increase in viral RNAs, proteins and secreted DNA that was observed in both PHH and dHepaRG (Fig 4 and S3 Fig). These results suggest that SRSF10 may normally repress the production of viral RNAs. While our results show that HBc and SRSF10 interact with each other, the role of this interaction in the regulation of the overall viral RNA amount remains unclear. In particular, 
future studies should be performed to decipher the role of $\mathrm{HBc}$ in the nuclear biogenesis of viral RNAs and to determine if and how it may counteract SRSF10 restrictive activity. The importance of SRSF10 in the HBV life cycle was further suggested by the finding that compound $1 C 8$, previously characterized as an inhibitor of SRSF10 phosphorylation [41], inhibited HBV replication by inducing a marked reduction of viral RNA levels in both HBV-infected dHepaRG cells and PHH (Fig 5). The opposite effects observed upon SRSF10 depletion and $1 \mathrm{C} 8$ treatment strongly suggest that the de-phosphorylated form of SRSF10 is responsible for the restriction effect. Accordingly, we confirmed that 1C8 equally induced SRSF10 de-phosphorylation in dHepaRG cells (S7 Fig). Following to this hypothesis, while treatment with 1C8 results in the accumulation of de-phosphorylated SRSF10, leading to a strong inhibitory phenotype, depletion of SRSF10 using siRNA affects all SRSF10 forms whether phosphorylated or not, thereby attenuating its restrictive activity. The involvement of SRSF10 in 1C8 antiviral effect is further suggested by the finding that it could be partially reverted upon SRSF10 depletion. As previously shown during AS, de-phosphorylation of SRSF10 may change its interaction with other cellular proteins important for HBV life cycle as well with HBc finally affecting its capacity to bind viral RNA [39,41]. It is also possible that 1C8 prevents the phosphorylation of other targets in addition to SRSF10. In particular, 1C8 may interfere with the phosphorylation of $\mathrm{HBc}$ in the nucleus, with consequences on its capacity to bind not only to other factors, in particular RBPs, but also DNA and/or RNA. Phosphorylation of $\mathrm{HBc}$ at serine residues within its CTD has been described as an important event to regulate pgRNA packaging and reverse transcription during nucleocapsid formation in the cytoplasm [17,65-69]. An attractive hypothesis is that phosphorylation of the nuclear pool of $\mathrm{HBc}$ may similarly control its capacity to bind to DNA and/or RNA by modulating its positive charges, as previously demonstrated by studies performed in bacteria [53]. The identification of kinase(s) inhibited by 1C8 will be critical to further decipher its mode of action and develop new, more efficient and selective compounds that could be used in combination with other already in use anti-viral drugs (such as NUC or CAM) to induce a long-lasting inhibition of viral replication. Of course, this perspective should first tackle the potential toxicity issue raised by using a host-targeting agent.

Finally, one surprising finding of our study is that neither SRSF10 nor 1C8 altered the splicing of HBV RNAs, as compared to what observed for other viruses (S10 Fig) [41,70]. While this result does not exclude that part of their effects on HBV may be indirectly mediated by an alteration in splicing of other cellular RNAs, our data rather suggest that both SRSF10 and 1C8 may act by controlling the level of nascent HBV RNAs (Fig 7). Beside their canonical role during splicing, several SR proteins were found to act at several other steps of cellular and viral RNA metabolism including transcription, stability and nuclear egress [32,71,72]. Notably, besides pre-mRNA splicing, some SR proteins have been reported to directly or indirectly associate to the phosphorylated CTD of RNA polymerase II and to stimulate transcriptional elongation [73,74]. Interestingly, several RBPs previously identified as important for HBV replication were shown to participate in the control of HBV transcription and/or in RNA stability. This is the case in particular for the splicing factors HNRNPC, HNRNPK, PUF60, RBM24 and TARBP [75-79]. It is therefore conceivable that SRSF10 may associate with HBV nascent RNA in the nucleus to control its synthesis/elongation and/or its stability. Recent studies have indicated that HBV RNA stability is controlled by m-6A methylation [80,81]. SRSF10 may intervene in HBV RNA stability by interacting with $\mathrm{m}-6 \mathrm{~A}$ readers or interfering with their RNA binding activity [82]. Future studies analyzing epigenetic changes and modifications in $\mathrm{HBc} /$ SRSF10 cccDNA/RNA binding activities in the presence of 1C8 should help uncover the underlying mechanisms. 
Altogether, our study revealed that nuclear $\mathrm{HBc}$ can connect to an array of cellular RBPs. It also identified SRSF10 as a restriction factor in HBV viral RNA production, therefore providing a basis for the evaluation of a new class of host-targeted antiviral compounds that could improve the current anti-HBV arsenal and encourage combinational therapies.

\section{Methods}

\section{Ethics statement}

All animal experiments were performed in accordance with the European Union guidelines for approval of the protocols by the local ethics committee (Authorization Agreement C2EA15, "Comité Rhône-Alpes d'Ethique pour l'Expérimentation Animale", Lyon, France-APAFIS\# 1570-2015073112163380 v4).

Primary human hepatocytes $(\mathrm{PHH})$ were freshly prepared from human liver resections obtained with a formal written consent from the donors, at the Centre Léon Bérard (Lyon) with French ministerial authorizations (AC 2013-1871, DC 2013-1870, AFNOR NF 96900 sept 2011).

\section{Cell culture and HBV infection}

HepaRG cells were cultured, differentiated, and infected by HBV as previously described [34]. HepaRG-TR-HBc and HepaRG-TR-ST-HBc were obtained by transducing HepaRG-TR cells [83] with Lenti4/TO lentiviral vectors expressing either $\mathrm{HBc}$ or ST-HBc under the control of the minimal CMV/TetOn promoter. Details on sequences are available upon request. Transduced cells were selected using blasticin $(10 \mu \mathrm{g} / \mathrm{mL})$ and zeocyn $(100 \mu \mathrm{g} / \mathrm{mL})$, then amplified and frozen as polyclonal lines. Primary human hepatocytes (PHH) were freshly prepared from human liver resection as previously described [84]. HBV genotype D inoculum (subtype ayw) was prepared from HepAD38 [85] cell supernatant by polyethylene-glycol-MW-8000 (PEG8000, SIGMA) precipitation (8\% final) as previously described [86]. Other HBV genotype viral inocula were similarly prepared from the supernatant of a newly developed stably-transformed HepG2 cell lines. Briefly, cell lines were obtained by transfection of a linearized pcDNA3Neo-HBV plasmid containing 1.35 genome unit of a consensus sequence of $\mathrm{HBV}$ genotype A, B, C, E, G, and H (obtained from HBV database: https://hbvdb.lyon.inserm.fr/; ref [87], the sequences are available upon request) and a double-round selection under G418 (500 $\mathrm{ng} / \mathrm{mL}$ ) by colony cell cloning (very low density seeding in large flasks). The titer of endotoxin free viral stocks was determined by qPCR. Cells were infected overnight in a media supplemented with $4 \%$ final of PEG, as previously described [34]. Infection was verified by measuring secreted HBsAg and HBeAg 7 to 10 days later by CLIA (Chemo-Luminescent Immune Assay) following manufacturer's instructions (AutoBio, China).

\section{Production of AAV_HBV vectors}

The AAV-HBVwt plasmid was obtained from ML Michel (Pasteur Institute) [88]. It contains the 1.2 genome copies of HBV genotype D sequence inserted between the AAV2 inverted terminal repeats (ITRs). The AAV-HBVnoHBc and AAV-HBV $\triangle \mathrm{HBc}$ vector plasmids were generated by inserting 1.3 genome units of a mutated HBV genotype $\mathrm{D}$ sequence between the AAV2 ITRs. The AAV-HBVnoHBc vector has a mutation in the HBc ATG (ATG to ATT). The AAV-HBV $\triangle \mathrm{HBc}$ has a deletion of the first 406 nucleotides of the $\mathrm{HBc}$ coding sequence. AAV serotype 3 vectors were produced by transient transfection of 293 cells and double purification on $\mathrm{CsCl}$ gradients by the Vector Core of the University of Nantes as previously described [89]. Vectors were titrated by qPCR using primers on the AAV ITRs. 


\section{Chemical reagents}

Unless otherwise specified, chemical reagents, drugs, antibiotics were purchased from Sigma Aldrich. Tenofovir (TDF) was a kind gift of Gilead Sciences (Foster city, USA). The core assembly modulator (CAM) used in the experiments was previously described [42], and resynthesized by AI-Biopharma (Montpellier, France). 1C8, i.e. 1C8, i.e. 1-Methyl-N-(5-nitrobenzo [d]isothiazol-3-yl)-4-oxo-1,4-dihydropyridine-3-carboxamide, was synthesized in Dr Gierson laboratory, but also re-synthesized and purified at $99 \%$ by AGV (Montpellier, France). RG7834 [90], a molecule that destabilizes HBV RNAs was synthetized by AI-Biopharma (Montpellier, France).

\section{Purification of ST-HBc complexes and sample preparation for mass spectrometry}

HepaRG-TR-HBc and HepaRG-TR-ST-HBc cells $\left(1,25 \times 10^{8}\right.$ cells $)$ were first differentiated, then transgene expression induced for 72 hours by adding Tet $(5 \mu \mathrm{g} / \mathrm{ml})$ in the culture media. Nuclei were purified from cells using the NE-PER Nuclear and Cytoplasmic Extraction kit (Thermo Scientific), and nuclear extracts prepared by suspending nuclei in NER solution in the presence of protease inhibitors (Complete EDTA-free Protease Inhibitor Cocktail, Roche) and digestion or not with Benzonase (Sigma-Aldrich), during $40 \mathrm{~min}$ on ice. Nuclear extract recovered after centrifugation were added on Strep-Tactin gravity columns (IBA) and further purified following manufacturer's instructions.

\section{Capsid migration assay}

The intracellular formation of HBV nucleocapsids was assessed by native agarose gel electrophoresis of cell lysates, followed by transfer onto the enhanced chemiluminescence membrane and western blot analysis, as described previously [91].

\section{Mass spectrometry-based quantitative proteomic analyses}

Eluted proteins were stacked in a single band in the top of a SDS-PAGE gel (4-12\% NuPAGE, Life Technologies) and stained with Coomassie blue R-250 before in-gel digestion using modified trypsin (Promega, sequencing grade) as previously described [92]. Resulting peptides were analyzed by online nanoliquid chromatography coupled to tandem MS (UltiMate 3000 and LTQ-Orbitrap Velos Pro, Thermo Scientific). Peptides were sampled on a $300 \mu \mathrm{m}$ x $5 \mathrm{~mm}$ PepMap C18 precolumn and separated on a $75 \mu \mathrm{m} \times 250 \mathrm{~mm}$ C18 column (PepMap, Thermo Scientific) using a 120-min gradient. MS and MS/MS data were acquired using Xcalibur (Thermo Scientific).

Peptides and proteins were identified and quantified using MaxQuant (version 1.5.3.30) [93] using the Uniprot database (Homo sapiens taxonomy proteome, October 2016 version), the sequence of $\mathrm{HBc}$, and the frequently observed contaminant database embedded in MaxQuant. Trypsin was chosen as the enzyme and 2 missed cleavages were allowed. Peptide modifications allowed during the search were: carbamidomethylation (C, fixed), acetyl (Protein Nter, variable) and oxidation ( $M$, variable). Minimum peptide length was set to 7 amino acids. Minimum number of peptides, razor + unique peptides and unique peptides were set to 1 . Maximum false discovery rates-calculated by employing a reverse database strategy-were set to 0.01 at PSM and protein levels. The "match between runs" option was activated. The iBAQ value [94] calculated by MaxQuant using razor + unique peptides was used to quantify proteins.

Statistical analysis were performed using ProStaR [95]. Proteins identified in the reverse and contaminant databases and proteins exhibiting less than 3 quantification values in one 
condition were discarded from the list. After $\log 2$ transformation, iBAQ values were normalized by median centering before missing value imputation (2.5-percentile value of each sample). Statistical testing was conducted using limma test. Differentially-expressed proteins were sorted out using a $\log _{2}$ (fold change) cut-off of 2 and a p-value cut-off allowing to reach a FDR inferior to $1 \%$ according to the Benjamini-Hochberg procedure ( $\mathrm{p}<0.0079$ and $\mathrm{p}<0.0063$ for dataset with and without Benzonase, respectively).

\section{Gene Ontology analyses and construction of the $\mathrm{HBc}$-interactome}

Proteomics data from the two conditions (without (benz-) or with (benz+) benzonase) were filtered out according to p-value $(<0.005)$ and fold change $(>4)$. Significant proteins common to the two conditions were extracted with a Venn diagram using their UniProtKB accession numbers. Statistical overrepresentation tests of these proteins were computed with PantherDB 11.1 and GO complete annotation sets. Overrepresented protein accession numbers were selected to further build and analyze their interacting network by means of Cytoscape software 3.5.1, querying IntAct molecular interaction database (May 27, 2017) with PSICQUIC service application 3.3.1, and Network Analyzer application 3.3.2.

\section{siRNA transfection}

dHepaRG or PHH cells seeded into a 24-well plate were transfected with $25 \mathrm{nM}$ or $10 \mathrm{nM}$ of siRNA using Dharmafect\#1 (GE HealthCare) or Lipofectamine RNAiMax (Life Technologies), respectively, following manufacturer's instructions. SiRNA used were the following: siSRSF10 (Dharmacon SmartPool L-190401), siRBMX (Dharmacon SmartPool L-011691), siControl (Dharmacon D-001810).

\section{In vivo experiments}

Primary human hepatocytes (PHH, Corning, BD Gentest or biopredic) were intrasplenically injected in NODFRG mice, a triple mutant mouse knocked-out for fumarylacetoacetate hydrolase (fah-/-), recombinase activating gene 2 (rag2-/-), interleukin 2 receptor gamma chain (IL2rg-/-). 48h after adeno-uPA conditioning [96], mice were subjected to NTBC (Swedish Orphan Biovitrum) cycling during the liver repopulation process, as described previously [97]. Mice with human serum albumin (HSA) levels $>15 \mathrm{mg} / \mathrm{mL}$, as determined using a Cobas C501 analyzer (Roche Applied Science), were inoculated with virus preparations by intra-peritoneal injection. Sera were collected at different time points before and after infection. Mice were sacrificed 10 weeks post-infection and liver sections snap frozen.

\section{Co-immunoprecipitation and western blot analysis}

For Co-IP analyses, 300-500 $\mu$ g of nuclear extracts, prepared as indicated above either from dHepaRG cells or from frozen liver sections were precleared with Protein A/G magnetic beads (Pierce) for $2 \mathrm{hrs}$ at $40^{\circ} \mathrm{C}$ and then incubated over-night at $4^{\circ} \mathrm{C}$ on a rotating wheel with $2 \mu \mathrm{g}$ of anti $\mathrm{HBc}$ antibody (Dako B0586) or home generated anti-HBc (1/40000; generous gift from $\mathrm{Dr}$ Adam Zlotnick, Bloomington, USA). Immune-complexes were captured with protein A/G magnetic beads, washed four times in IP buffer and then eluted by boiling for $5 \mathrm{~min}$ in $2 \mathrm{X}$ loading buffer (Laemmli). For western blot, proteins were resolved by SDS-PAGE and then transferred onto a nitrocellulose membrane. Membranes were incubated with the primary antibodies corresponding to the indicated proteins. Proteins were revealed by chemi-luminescence (Super Signal West Dura Substrate, Pierce) using a secondary peroxydase-conjugated antibody (Dako) at a dilution of 1:10000. Primary antibodies used were: anti-HBc (Ab140243, 
1/1000) or a home generated anti-HBc (1/40000; generous gift from Dr Adam Zlotnick, Bloomington, USA), anti-SRSF10 (Ab77209, 1/2000), anti-RBMX (Ab190352, 1/2000), antiTRA2B (Ab171082, 1/2000), anti-SRSF1 (Ab38017, 1/1000), anti-SRSF2 (Ab204916, 1/1000), anti-DDX17 (Proteintech 19910-1-AP, 1/1000), anti-PARP1 (Ab6079, 1/1000), anti-DNAJB6 (Ab198995, 1/1000), anti- $\beta$-Tubulin (Ab6044, 1/10000), anti-NTCP (Ab131084, 1/1000), antiLamin B1 (Ab16048, 1/10000).

\section{Two-dimensional gel electrophoresis}

For two-Dimensional (2D) gel electrophoresis $150 \mu \mathrm{g}$ of nuclear proteins were dissolved in $150 \mu 1$ of 2D DeStreak Rehydration Solution and 0,5\% IPG buffer (GE Healthcare). Samples were loaded on immobilized pH gradient Immobiline DryStrip gels pH 4-7 (GE Healthcare), isoelectrofocused with the Ettan IPGphor 3 Isoelectric Focusing System (GE Healthcare) according to the manufacturer's instructions. The IPG strips were layered onto a 8-16\% Criterion TGX Stain-Free Protein Gel (BIORAD). SDS-PAGE was performed with a Criterion cell (BIORAD) and blotted onto nitrocellulose membranes with Trans-Blot Turbo Transfer System (BIORAD) as recommended by the manufacturer. Membranes were then incubated with anti-SRSF10 antibody.

\section{Immunofluorescence analyses}

Analyses were performed as described previously using anti-HBc (Thermo MA1-7607, 1/500) primary antibody and Alexa Fluor 555 secondary antibodies (Molecular Probes) [92]. Nuclei were stained with Hoescht 33258. Images were collected on a confocal NLO-LSM 880 microscope (Zeiss). Further image processing was performed using ICY [98].

\section{Nucleic acid extractions and analysis}

Total RNA and DNA were extracted from cells with the NucleoSpin RNA II and Nucleospin 96 tissue kit, respectively, according to the manufacturer's instructions (Macherey-Nagel). RNA reverse transcription was performed using SuperScript III (Invitrogen). Quantitative PCR for HBV were performed using HBV specific primers and normalized to PRNP housekeeping gene as previously described [99]. Pre-genomic RNA was quantified using the TaqMan Fast Advanced Master Mix (Life Technologies) and normalized to GusB cDNA levels. $\mathrm{HBV}$ cccDNA was quantified from total DNA following digestion for $45 \mathrm{~min}$ at $37^{\circ} \mathrm{C}$ with T5 exonuclease (Epicentre) to remove rcDNA followed by $30 \mathrm{~min}$ heat inactivation. cccDNA amount was quantified by TaqMan qPCR analyses and normalized to $\beta$-globin cDNA level, as previously described [100]. Analysis of HBV RNAs by Northern blot was performed as previously described [101]

\section{Analysis of spliced HBV RNA}

The analysis of HBV spliced RNA was performed the RNomics platform of the University of Sherbrooke (Canada) as previously described [102,103]. After reverse-transcription, quantitative qPCR was performed using primers designed to detect each spliced and unspliced RNA and normalized to the MLRP19, PUM1 et YWHAZ genes (S2 Table). Primers were designed to detect 15 spliced RNA (sv1 to sv15) and 3 intronic regions (intron 1,2 and 2b), as described in ref. [10]. 


\section{Quantification of nascent HBV RNA}

HBV nascent RNA were quantified using the Click-iT Nascent RNA Capture Kit (Life Technologies) following the manufacturer's instructions. Briefly HBV-infected dHepaRG were incubated for 2 hours with 5-ethynyl Uridine (EU) before RNA extraction and biotinylation. Control was provided by cells treated with Actinomycin D $(1 \mu \mathrm{M}) 20 \mathrm{~min}$ before labeling. Biotinylated RNA was purified on streptavidin magnetic beads. Total and EU-labeled RNA was reverse-transcribed and quantified as indicated above.

\section{Viability/cytotoxicity assays}

Viability/cytotoxicity was assessed using the CellTiter-Glo Luminiscent assay (Promega) following the manufacturer's instructions.

\section{Statistical analysis}

Statistical analyses were performed using the XLStat software and Kruskal-Wallis tests with multiple comparison respect to non-treated cells (Dunn's post-test). For all tests, a $\mathrm{p}$ value $\leq 0,05$ was considered as significant. ${ }^{*}$ correspond to $\mathrm{p}$ value $\leq 0.05$; ${ }^{* *}$ correspond to $\mathrm{p}$ value $\leq 0.01 ;{ }^{* * *}$ correspond to $\mathrm{p}$ value $\leq 0.001$.

\section{Supporting information}

S1 Fig. Functional analysis of dHepaRG-TR-ST-HBc cells. (A) Immunofluorescence (IF) of analysis of HBc localization in dHepaRG-TR-ST-HBc versus HBV-infected dHepaRG and PHH. (B) Intracellular HBV capsids, produced by the indicated cell lines, were analyzed by native gel electrophoresis followed by western blot with anti-HBc antibody. Lanes: 1. HepG2.2.15 2. dHepaRG-TR-HBe; 3. dHepaRG-TR-HBc; 4. dHepaRG-TR-ST-HBc. (TIF)

S2 Fig. Structure of SRSF10 isoforms detected in anti-HBc IP assays. (A) Structure of the two major SRSF10 isoforms. These two SRSF10 variants that migrate at 37 and 20-22 KDa correspond to the two major SRSF10 isoforms detected by the anti-SRSF10 antibody (Ab77209). Occasionally additional bands with an intermediate size are visible as shown in panel B. The red lines correspond to the regions targeted by the siRNA. (B) HBc was immune-precipitated from nuclear extracts of dHepaRG-HBc (HBc), dHepaRG-ST-HBc (ST-HBc) and control dHepaRG (RG) cells induced with Tet for two days. Eluted proteins were analyzed by western blot using anti-HBc and anti-SRSF10 antibodies. The asterisk indicates the positions of IgG heavy chain. (C) Putative SRSF10 isoform migrating between 25 and $35 \mathrm{KDa}$.

S3 Fig. Effect of SRSF10 or RBMX KD on HBV replication in dHepaRG cells. (A) Outline of the experimental protocol in dHepaRG cells: cells were transfected with siRNA targeting SRSF10 or RBMX or control siRNA (siCTL) and then infected with HBV (MOI of 250 vge/ cell). (B) NTCP levels in siRNA transfected dHepaRG cells before HBV infection (D0). C. Western blot validations in cells secreted parameters measured at D7pi. Results are expressed as the mean normalized ratio $+/-\mathrm{SD}$, between siSRSF10 or siRBMX and siCTL transfected cells, of 3 independent experiments, each performed in triplicate.

S4 Fig. Northern blot analysis. dHepaRG cells were transfected CTL or SRSF10 siRNA and infected with HBV as previously described (S3A Fig). Total RNA was extracted from cells at 
D7 pi and analyzed by Northern blot using HBV probes. (TIF)

S5 Fig. Effect of SRSF10 on established HBV replication. (A) Outline of the experimental protocol: dHepaRG cells were infected with HBV (MOI of $250 \mathrm{vge} / \mathrm{cell}$ ) and then transfected twice with siRNA targeting SRSF10 or control siRNA (siCTL). Cells and supernatants were harvested at D15pi and analyzed to measure extracellular and intracellular HBV parameters. (B) Western blot validation of SRSF10 KD. (C) Effect of SRSF10 KD on intracellular and secreted HBV parameters. Results are expressed as the mean normalized ratio $+/$ - SD, between siSRSF10 or siRBMX and siCTL transfected cells, of 3 independent experiments, each performed in triplicate.

S6 Fig. Effect of SRSF10 KD on HBV RNAs produced in the absence of HBc. (A) dHepaRG cells were transfected with siRNA against SRSF10 or control siRNA and then transduced with $A A V$ vectors containing either a wt (AAVHBVwt) or an HBc-deficient genome (AAVHBVnoHBc and AAVHBV $\triangle \mathrm{HBc}$ ) at a MOI of $10^{4} \mathrm{vge} / \mathrm{cell}$. Secreted antigens and total RNAs were quantified 10 days later. Results are expressed as the mean normalized ratio $+/-\mathrm{SD}$, between siSRSF10 and siCTL transfected cells, of 3 independent experiments, each performed in triplicate.

(TIF)

S7 Fig. Analysis of SRSF10 phosphorylation by 2D-gel electrophoresis. Nuclear extracts were prepared from dHepaRG cells either mock (A) or 1C8-treated (18 hrs at $20 \mu \mathrm{M})(\mathrm{B})$ and separated by two-dimensional gel electrophoresis followed by western blot using an antiSRSF10 antibody. Numbers on the top of the images indicate the $\mathrm{pH}$ gradient. Only the larger SRSF10 isoform of $37 \mathrm{KDa}$ was visible under these conditions. The arrow indicates a hypophosphorylated isoform generated following $1 \mathrm{C} 8$ treatment. (TIF)

S8 Fig. Characterization of 1C8 EC50 and toxicity assay. (A) to (D). Measure of 1C8 EC50 on HBV-infected dHepaRG. dHepaRG cells were infected with HBV (MOI of 250 vge/cell) for 7 days followed by three treatments with increasing concentration of 1C8. Total HBV RNAs (A), secreted HBV DNA (B), HBsAg (C) and HBeAg (D) were measured two days after the last treatment. Results are presented as the mean change in expression or secretion $+/$ - SD of three independent experiments, each performed in triplicate. (E) Toxicity assay. Cell viability of dHepaRG cells treated with increasing concentrations of $1 \mathrm{C} 8$, was measured using the CellTiter-Glo Luminiscent Cell Viability Assay (Promega). Non-infected (NI) and HBV-infected dHepaRG cells treated with DMSO and puromycin were used as negative and positive controls, respectively.

S9 Fig. Effect of $1 \mathrm{C8}$ on the replication of various HBV genotypes in dHepaRG cells. (A) Cells were infected with HBV genotype C (MOI of 100 vge/cell) and treated as indicated in Fig 5A. Treatments included, Tenofovir (TDF at $10 \mu \mathrm{M}$ ), a Core allosteric modulator (CAM at $10 \mu \mathrm{M})$ or $1 \mathrm{C} 8(10 \mu \mathrm{M})$. Intracellular and secreted HBV parameters were quantified 2 days after the last treatment. Results are expressed as the mean normalized ratio +/- SD between non-treated and treated cells of 2 independent experiments, each performed in triplicate. (B) Cells were infected with indicated HBV genotypes (MOI of 100 vge/cell) and either mock- or treated with $1 \mathrm{C} 8(10 \mu \mathrm{M})$. HBeAg and HBsAg were quantified by CLIA. Results are expressed as the mean normalized ratio $+/$ - SD between non-treated and treated cells of a single 
experiment, with biological triplicates.

(TIF)

S10 Fig. Analysis of spliced and unspliced HBV RNA following SRSF10 KD or 1C8 treatment. (A) and (B) Total RNA were extracted from dHepaRG (A) and PHH (B) transfected with siRNA following the previously described protocol (Fig 4A). (C) HBV-infected dHepaRG were treated with $1 \mathrm{C} 8$ as previously described (Fig 5B). HBV RNAs were analyzed by endpoint RT-qPCR using sets of primers able to discriminate each spliced and unspliced form (see Methods section). Results are expressed as the mean ratio +/- SD between siSRSF10 and siCTL transfected cells of 3(A and C) or 2 (B) independent experiments.

S1 Table. HBc interactome analysis using MS-based quantitative proteomics. ST-HBc-associated proteins were identified using MS-based proteomics. For this, cell lysate expressing $\mathrm{ST}-\mathrm{HBc}$ or untagged $\mathrm{HBc}$ were treated or not with Benzonase before purification by affinity using Strep-Tactin. Eluted proteins were digested with trypsin and the resulting peptides submitted to MS-based proteomic analysis. The proteins were then identified and quantified in each sample before statistical analysis allowing to sort out proteins enriched with ST-HBc compared to untagged $\mathrm{HBc}$ used as negative control.

(XLSX)

S2 Table. List of primers used for the quantification of spliced and unspliced HBV RNAs. (XLSX)

\section{Acknowledgments}

We would like to thank Adam Zlotnick for providing the anti-HBc antibody, Christophe Vanbelle (Imaging platform of CRCL) for his help on confocal microscope analyses, Brieux Chardès and Claire Bugnot for technical assistance, Laura Dimier, Jennifer Molle, Océane Floriot and Anaëlle Dubois for their help with the isolation of primary human hepatocytes, as well as the staff from Pr. Michel Rivoire's surgery room for providing us with liver resections. We also thank the Plateforme de Thérapie Génique in Nantes (France) for the production of the in vivo certified lots of adeno-uPA vector and AAV vectors, Jean-François Henry, Nadine Aguilera and Tiphaine Dorel from the animal facility (PBES, Plateau de Biologie Experimental de la Souris, UMS3444/CNRS, US8/Inserm, ENS de Lyon, UCBL) and Veronique Pierre for her technical help in handling of mice. We also thank Philippe Thibault, Elvy Lapointe and Mathieu Durand at the RNA platform of Université de Sherbrooke.

\section{Author Contributions}

Conceptualization: Benoit Chabot, Julie Lucifora, David Durantel, Anna Salvetti.

Data curation: Yohann Couté, Lucid Belmudes, Yujin Kim, Christophe Combet.

Formal analysis: Hélène Chabrolles, Yujin Kim.

Funding acquisition: Fabien Zoulim, David Durantel, Anna Salvetti.

Investigation: Hélène Chabrolles, Héloïse Auclair, Serena Vegna, Thomas Lahlali, Yohann Couté, Lucid Belmudes, Gilliane Chadeuf, Pascal Jalaguier, Christophe Combet, Benoit Chabot, Julie Lucifora, David Durantel.

Methodology: Hélène Chabrolles, Hélö̈se Auclair, Serena Vegna, Caroline Pons, Maud Michelet, Yohann Couté, Ariel Di Bernardo, Pascal Jalaguier, Floriane Fusil. 
Resources: François-Loïc Cosset, Michel Rivoire, Lee D. Arnold, Uri Lopatin, David Grierson.

Writing - original draft: Hélène Chabrolles.

Writing - review \& editing: Fabien Zoulim, David Grierson, Benoit Chabot, Julie Lucifora, David Durantel, Anna Salvetti.

\section{References}

1. Levrero M, Zucman-Rossi J. Mechanisms of HBV-induced hepatocellular carcinoma. J Hepatol. 2016; 64(1 Suppl):S84-101. https://doi.org/10.1016/j.jhep.2016.02.021 PMID: 27084040.

2. Zoulim F, Durantel D. Antiviral therapies and prospects for a cure of chronic hepatitis B. Cold Spring Harb Perspect Med. 2015; 5(4). https://doi.org/10.1101/cshperspect.a021501 PMID: 25833942.

3. Zeisel MB, Lucifora J, Mason WS, Sureau C, Beck J, Levrero M, et al. Towards an HBV cure: state-ofthe-art and unresolved questions - report of the ANRS workshop on HBV cure. Gut. 2015; 64 (8):1314-26. https://doi.org/10.1136/gutjnl-2014-308943 PMID: 25670809.

4. Durantel D, Zoulim F. New antiviral targets for innovative treatment concepts for hepatitis $B$ virus and hepatitis delta virus. J Hepatol. 2016; 64(1 Suppl):S117-31. https://doi.org/10.1016/j.jhep.2016.02. 016 PMID: 27084032.

5. Fanning GC, Zoulim F, Hou J, Bertoletti A. Therapeutic strategies for hepatitis B virus infection: towards a cure. Nat Rev Drug Discov. 2019. Epub 2019/08/29. https://doi.org/10.1038/s41573-0190037-0 PMID: 31455905.

6. Seeger C, Mason WS. Molecular biology of hepatitis B virus infection. Virology. 2015; 479-480:67286. https://doi.org/10.1016/j.virol.2015.02.031 PMID: 25759099; PubMed Central PMCID: PMC4424072.

7. Koniger C, Wingert I, Marsmann M, Rosler C, Beck J, Nassal M. Involvement of the host DNA-repair enzyme TDP2 in formation of the covalently closed circular DNA persistence reservoir of hepatitis B viruses. Proc Natl Acad Sci U S A. 2014; 111(40):E4244-53. Epub 2014/09/10. https://doi.org/10. 1073/pnas.1409986111 PMID: 25201958; PubMed Central PMCID: PMC4209993.

8. Nassal M. HBV cccDNA: viral persistence reservoir and key obstacle for a cure of chronic hepatitis B. Gut. 2015; 64(12):1972-84. https://doi.org/10.1136/gutjnl-2015-309809 PMID: 26048673.

9. Wei L, Ploss A. Core components of DNA lagging strand synthesis machinery are essential for hepatitis B virus cccDNA formation. Nat Microbiol. 2020. Epub 2020/03/11. https://doi.org/10.1038/s41564020-0678-0 PMID: 32152586.

10. Sommer G, Heise T. Posttranscriptional control of HBV gene expression. Front Biosci. 2008; 13:5533-47. PubMed https://doi.org/10.2741/3097 PMID: 18508603.

11. Redelsperger F, Lekbaby B, Mandouri Y, Giang E, Duriez M, Desire N, et al. Production of hepatitis B defective particles is dependent on liver status. Virology. 2012; 431(1-2):21-8. Epub 2012/06/06. https://doi.org/10.1016/j.virol.2012.05.008 PMID: 22664356.

12. Zlotnick A, Venkatakrishnan B, Tan Z, Lewellyn E, Turner W, Francis S. Core protein: A pleiotropic keystone in the HBV lifecycle. Antiviral Res. 2015; 121:82-93. https://doi.org/10.1016/j.antiviral.2015. 06.020 PMID: 26129969; PubMed Central PMCID: PMC4537649.

13. Chu TH, Liou AT, Su PY, Wu HN, Shih C. Nucleic acid chaperone activity associated with the argininerich domain of human hepatitis B virus core protein. J Virol. 2014; 88(5):2530-43. Epub 2013/12/20. https://doi.org/10.1128/JVI.03235-13 PMID: 24352445; PubMed Central PMCID: PMC3958103.

14. Li HC, Huang EY, Su PY, Wu SY, Yang CC, Lin YS, et al. Nuclear export and import of human hepatitis B virus capsid protein and particles. PLoS Pathog. 2010; 6(10):e1001162. Epub 2010/11/10. https:// doi.org/10.1371/journal.ppat.1001162 PMID: 21060813; PubMed Central PMCID: PMC2965763.

15. Liu K, Ludgate L, Yuan Z, Hu J. Regulation of multiple stages of hepadnavirus replication by the carboxyl-terminal domain of viral core protein in trans. J Virol. 2015; 89(5):2918-30. Epub 2014/12/30. https://doi.org/10.1128/JVI.03116-14 PMID: 25540387; PubMed Central PMCID: PMC4325754.

16. Nassal $M$. The arginine-rich domain of the hepatitis $B$ virus core protein is required for pregenome encapsidation and productive viral positive-strand DNA synthesis but not for virus assembly. J Virol. 1992; 66(7):4107-16. Epub 1992/07/01. PubMed https://doi.org/10.1128/JVI.66.7.4107-4116.1992 PMID: 1602535; PubMed Central PMCID: PMC241213.

17. Diab A, Foca A, Zoulim F, Durantel D, Andrisani O. The diverse functions of the hepatitis B core/capsid protein $(\mathrm{HBC})$ in the viral life cycle: Implications for the development of HBc-targeting antivirals. Antiviral Res. 2018; 149:211-20. https://doi.org/10.1016/j.antiviral.2017.11.015 PMID: 29183719; PubMed Central PMCID: PMC5757518. 
18. Rabe B, Delaleau M, Bischof A, Foss M, Sominskaya I, Pumpens $P$, et al. Nuclear entry of hepatitis $B$ virus capsids involves disintegration to protein dimers followed by nuclear reassociation to capsids. PLoS Pathog. 2009; 5(8):e1000563. https://doi.org/10.1371/journal.ppat.1000563 PMID: 19714236; PubMed Central PMCID: PMC2727048.

19. Blondot ML, Bruss V, Kann M. Intracellular transport and egress of hepatitis B virus. J Hepatol. 2016; 64(1 Suppl):S49-59. https://doi.org/10.1016/j.jhep.2016.02.008 PMID: 27084037.

20. Akiba $T$, Nakayama $H$, Miyazaki $Y$, Kanno A, Ishii M, Ohori $H$. Relationship between the replication of hepatitis $B$ virus and the localization of virus nucleocapsid antigen (HBcAg) in hepatocytes. J Gen Virol. 1987; 68 (Pt 3):871-7. Epub 1987/03/01. https://doi.org/10.1099/0022-1317-68-3-871 PMID: 3819701.

21. Deroubaix A, Osseman Q, Cassany A, Begu D, Ragues J, Kassab S, et al. Expression of viral polymerase and phosphorylation of core protein determine core and capsid localization of the human hepatitis B virus. J Gen Virol. 2015; 96(Pt 1):183-95. Epub 2014/10/03. https://doi.org/10.1099/vir.0. 064816-0 PMID: 25274856.

22. Guidotti LG, Martinez V, Loh YT, Rogler CE, Chisari FV. Hepatitis B virus nucleocapsid particles do not cross the hepatocyte nuclear membrane in transgenic mice. J Virol. 1994; 68(9):5469-75. Epub 1994/09/01. PubMed https://doi.org/10.1128/JVI.68.9.5469-5475.1994 PMID: 8057429; PubMed Central PMCID: PMC236947.

23. Zhang $X, L u$ W, Zheng $Y$, Wang W, Bai L, Chen L, et al. In situ analysis of intrahepatic virological events in chronic hepatitis B virus infection. J Clin Invest. 2016; 126(3):1079-92. https://doi.org/10. 1172/JCI83339 PMID: 26901811; PubMed Central PMCID: PMC4767362.

24. Bock CT, Schwinn S, Locarnini S, Fyfe J, Manns MP, Trautwein C, et al. Structural organization of the hepatitis B virus minichromosome. J Mol Biol. 2001; 307(1):183-96. https://doi.org/10.1006/jmbi. 2000.4481 PMID: 11243813.

25. Hatton T, Zhou S, Standring DN. RNA- and DNA-binding activities in hepatitis B virus capsid protein: a model for their roles in viral replication. J Virol. 1992; 66(9):5232-41. PubMed https://doi.org/10.1128/ JVI.66.9.5232-5241.1992 PMID: 1501273; PubMed Central PMCID: PMC289076.

26. Pollicino T, Belloni L, Raffa G, Pediconi N, Squadrito G, Raimondo G, et al. Hepatitis B virus replication is regulated by the acetylation status of hepatitis $B$ virus cccDNA-bound $\mathrm{H} 3$ and $\mathrm{H} 4$ histones. Gastroenterology. 2006; 130(3):823-37. Epub 2006/03/15. https://doi.org/10.1053/j.gastro.2006.01.001 PMID: 16530522

27. Guo YH, Li YN, Zhao JR, Zhang J, Yan Z. HBc binds to the CpG islands of HBV cccDNA and promotes an epigenetic permissive state. Epigenetics. 2011; 6(6):720-6. Epub 2011/05/07. https://doi.org/10. 4161/epi.6.6.15815 PMID: 21546797.

28. Chong CK, Cheng CYS, Tsoi SYJ, Huang FY, Liu F, Seto WK, et al. Role of hepatitis B core protein in HBV transcription and recruitment of histone acetyltransferases to cccDNA minichromosome. Antiviral Res. 2017; 144:1-7. Epub 2017/05/14. https://doi.org/10.1016/j.antiviral.2017.05.003 PMID: 28499864.

29. Guo Y, Kang W, Lei X, Li Y, Xiang A, Liu Y, et al. Hepatitis B viral core protein disrupts human host gene expression by binding to promoter regions. BMC Genomics. 2012; 13:563. https://doi.org/10. 1186/1471-2164-13-563 PMID: 23088787; PubMed Central PMCID: PMC3484065.

30. McGonigle R, Yap WB, Ong ST, Gatherer D, Bakker SE, Tan WS, et al. An N-terminal extension to the hepatitis $\mathrm{B}$ virus core protein forms a poorly ordered trimeric spike in assembled virus-like particles. Journal of structural biology. 2015; 189(2):73-80. Epub 2015/01/06. https://doi.org/10.1016/j.jsb. 2014.12.006 PMID: 25557498; PubMed Central PMCID: PMC4318616.

31. Long JC, Caceres JF. The SR protein family of splicing factors: master regulators of gene expression. Biochem J. 2009; 417(1):15-27. https://doi.org/10.1042/BJ20081501 PMID: 19061484.

32. Jeong S. SR Proteins: Binders, Regulators, and Connectors of RNA. Mol Cells. 2017; 40(1):1-9. Epub 2017/02/06. https://doi.org/10.14348/molcells.2017.2319 PMID: 28152302; PubMed Central PMCID: PMC5303883.

33. Twyffels L, Gueydan C, Kruys V. Shuttling SR proteins: more than splicing factors. FEBS J. 2011; 278 (18):3246-55. Epub 2011/07/29. https://doi.org/10.1111/j.1742-4658.2011.08274.x PMID: 21794093.

34. Gripon P, Rumin S, Urban S, Le Seyec J, Glaise D, Cannie I, et al. Infection of a human hepatoma cell line by hepatitis B virus. Proc Natl Acad Sci U S A. 2002; 99(24):15655-60. Epub 2002/11/15. https:// doi.org/10.1073/pnas.232137699 PMID: 12432097; PubMed Central PMCID: PMC137772.

35. Hantz O, Parent R, Durantel D, Gripon P, Guguen-Guillouzo C, Zoulim F. Persistence of the hepatitis $B$ virus covalently closed circular DNA in HepaRG human hepatocyte-like cells. J Gen Virol. 2009; 90 (Pt 1):127-35. https://doi.org/10.1099/vir.0.004861-0 PMID: 19088281.

36. Shin C, Manley JL. The SR protein SRp38 represses splicing in M phase cells. Cell. 2002; 111 (3):407-17. PubMed https://doi.org/10.1016/s0092-8674(02)01038-3 PMID: 12419250. 
37. Feng Y, Chen M, Manley JL. Phosphorylation switches the general splicing repressor SRp38 to a sequence-specific activator. Nat Struct Mol Biol. 2008; 15(10):1040-8. Epub 2008/09/17. https://doi. org/10.1038/nsmb.1485 PMID: 18794844; PubMed Central PMCID: PMC2668916.

38. Shi Y, Manley JL. A complex signaling pathway regulates SRp38 phosphorylation and pre-mRNA splicing in response to heat shock. Mol Cell. 2007; 28(1):79-90. https://doi.org/10.1016/j.molcel.2007. 08.028 PMID: 17936706 .

39. Shin C, Feng Y, Manley JL. Dephosphorylated SRp38 acts as a splicing repressor in response to heat shock. Nature. 2004; 427(6974):553-8. https://doi.org/10.1038/nature02288 PMID: 14765198.

40. Cheung PK, Horhant D, Bandy LE, Zamiri M, Rabea SM, Karagiosov SK, et al. A Parallel Synthesis Approach to the Identification of Novel Diheteroarylamide-Based Compounds Blocking HIV Replication: Potential Inhibitors of HIV-1 Pre-mRNA Alternative Splicing. J Med Chem. 2016; 59(5):1869-79. Epub 2016/02/16. https://doi.org/10.1021/acs.jmedchem.5b01357 PMID: 26878150.

41. Shkreta L, Blanchette M, Toutant J, Wilhelm E, Bell B, Story BA, et al. Modulation of the splicing regulatory function of SRSF10 by a novel compound that impairs HIV-1 replication. Nucleic Acids Res. 2017; 45(7):4051-67. https://doi.org/10.1093/nar/gkw1223 PMID: 27928057; PubMed Central PMCID: PMC5397194.

42. Lahlali T, Berke JM, Vergauwen K, Foca A, Vandyck K, Pauwels F, et al. Novel Potent Capsid Assembly Modulators Regulate Multiple Steps of the Hepatitis B Virus Life Cycle. Antimicrob Agents Chemother. 2018;62(10). Epub 2018/07/18. https://doi.org/10.1128/AAC.00835-18 PMID: 30012770; PubMed Central PMCID: PMC6153789.

43. Huang HL, Jeng KS, Hu CP, Tsai CH, Lo SJ, Chang C. Identification and characterization of a structural protein of hepatitis $B$ virus: a polymerase and surface fusion protein encoded by a spliced RNA. Virology. 2000; 275(2):398-410. Epub 2000/09/22. https://doi.org/10.1006/viro.2000.0478 PMID: 10998339

44. Soussan $P$, Garreau F, Zylberberg H, Ferray C, Brechot C, Kremsdorf D. In vivo expression of a new hepatitis B virus protein encoded by a spliced RNA. J Clin Invest. 2000; 105(1):55-60. Epub 2000/01/ 05. https://doi.org/10.1172/JCI8098 PMID: 10619861; PubMed Central PMCID: PMC382588.

45. Marion MJ, Hantz O, Durantel D. The HepaRG cell line: biological properties and relevance as a tool for cell biology, drug metabolism, and virology studies. Methods Mol Biol. 2010; 640:261-72. https:// doi.org/10.1007/978-1-60761-688-7_13 PMID: 20645056.

46. Hirose T, Nakagawa S. Paraspeckles: possible nuclear hubs by the RNA for the RNA. Biomol Concepts. 2012; 3(5):415-28. Epub 2012/10/01. https://doi.org/10.1515/bmc-2012-0017 PMID: 25436547.

47. Spector DL, Lamond AI. Nuclear speckles. Cold Spring Harb Perspect Biol. 2011; 3(2). Epub 2010/10/ 12. https://doi.org/10.1101/cshperspect.a000646 PMID: 20926517; PubMed Central PMCID: PMC3039535.

48. Chen $\mathrm{Y}$, Zhang $\mathrm{Y}$, Wang $\mathrm{Y}$, Zhang L, Brinkman EK, Adam SA, et al. Mapping 3D genome organization relative to nuclear compartments using TSA-Seq as a cytological ruler. J Cell Biol. 2018; 217 (11):4025-48. Epub 2018/08/30. https://doi.org/10.1083/jcb.201807108 PMID: 30154186; PubMed Central PMCID: PMC6219710.

49. Kim J, Venkata NC, Hernandez Gonzalez GA, Khanna N, Belmont AS. Gene expression amplification by nuclear speckle association. J Cell Biol. 2020; 219(1). Epub 2019/11/24. https://doi.org/10.1083/ jcb.201904046 PMID: 31757787.

50. Hentze MW, Castello A, Schwarzl T, Preiss T. A brave new world of RNA-binding proteins. Nat Rev Mol Cell Biol. 2018; 19(5):327-41. Epub 2018/01/18. https://doi.org/10.1038/nrm.2017.130 PMID: 29339797.

51. Ricco R, Kanduc D. Hepatitis B virus and Homo sapiens proteome-wide analysis: A profusion of viral peptide overlaps in neuron-specific human proteins. Biologics. 2010; 4:75-81. PubMed https://doi.org/ 10.2147/btt.s8890 PMID: 20531967; PubMed Central PMCID: PMC2880343.

52. Corley M, Burns MC, Yeo GW. How RNA-Binding Proteins Interact with RNA: Molecules and Mechanisms. Mol Cell. 2020; 78(1):9-29. Epub 2020/04/04. https://doi.org/10.1016/j.molcel.2020.03.011 PMID: 32243832.

53. Heger-Stevic J, Zimmermann P, Lecoq L, Bottcher B, Nassal M. Hepatitis B virus core protein phosphorylation: Identification of the SRPK1 target sites and impact of their occupancy on RNA binding and capsid structure. PLoS Pathog. 2018; 14(12):e1007488. https://doi.org/10.1371/journal.ppat. 1007488 PMID: 30566530; PubMed Central PMCID: PMC6317823.

54. Conrad T, Albrecht AS, de Melo Costa VR, Sauer S, Meierhofer D, Orom UA. Serial interactome capture of the human cell nucleus. Nat Commun. 2016; 7:11212. Epub 2016/04/05. https://doi.org/10. 1038/ncomms11212 PMID: 27040163; PubMed Central PMCID: PMC4822031. 
55. Adamson B, Smogorzewska A, Sigoillot FD, King RW, Elledge SJ. A genome-wide homologous recombination screen identifies the RNA-binding protein RBMX as a component of the DNA-damage response. Nat Cell Biol. 2012; 14(3):318-28. https://doi.org/10.1038/ncb2426 PMID: 22344029; PubMed Central PMCID: PMC3290715.

56. Matsunaga S, Takata H, Morimoto A, Hayashihara K, Higashi T, Akatsuchi K, et al. RBMX: a regulator for maintenance and centromeric protection of sister chromatid cohesion. Cell Rep. 2012; 1(4):299308. https://doi.org/10.1016/j.celrep.2012.02.005 PMID: 22832223.

57. Shin C, Kleiman FE, Manley JL. Multiple properties of the splicing repressor SRp38 distinguish it from typical SR proteins. Mol Cell Biol. 2005; 25(18):8334-43. https://doi.org/10.1128/MCB.25.18.83348343.2005 PMID: 16135820; PubMed Central PMCID: PMC1234314.

58. Shkreta L, Toutant J, Durand M, Manley JL, Chabot B. SRSF10 Connects DNA Damage to the Alternative Splicing of Transcripts Encoding Apoptosis, Cell-Cycle Control, and DNA Repair Factors. Cell Rep. 2016; 17(8):1990-2003. https://doi.org/10.1016/j.celrep.2016.10.071 PMID: 27851963; PubMed Central PMCID: PMC5483951.

59. Zhou X, Li X, Cheng Y, Wu W, Xie Z, Xi Q, et al. BCLAF1 and its splicing regulator SRSF10 regulate the tumorigenic potential of colon cancer cells. Nat Commun. 2014; 5:4581. https://doi.org/10.1038/ ncomms5581 PMID: 25091051.

60. Zhou X, Wu W, Li H, Cheng Y, Wei N, Zong J, et al. Transcriptome analysis of alternative splicing events regulated by SRSF10 reveals position-dependent splicing modulation. Nucleic Acids Res. 2014; 42(6):4019-30. https://doi.org/10.1093/nar/gkt1387 PMID: 24442672; PubMed Central PMCID: PMC3973337.

61. Hennig S, Kong G, Mannen T, Sadowska A, Kobelke S, Blythe A, et al. Prion-like domains in RNA binding proteins are essential for building subnuclear paraspeckles. J Cell Biol. 2015; 210(4):529-39. Epub 2015/08/19. https://doi.org/10.1083/jcb.201504117 PMID: 26283796; PubMed Central PMCID: PMC4539981.

62. An H, Tan JT, Shelkovnikova TA. Stress granules regulate stress-induced paraspeckle assembly. J Cell Biol. 2019; 218(12):4127-40. Epub 2019/10/23. https://doi.org/10.1083/jcb.201904098 PMID: 31636118; PubMed Central PMCID: PMC6891081.

63. Ninomiya K, Adachi S, Natsume T, Iwakiri J, Terai G, Asai K, et al. LncRNA-dependent nuclear stress bodies promote intron retention through SR protein phosphorylation. EMBO J. 2020; 39(3):e102729. Epub 2019/11/30. https://doi.org/10.15252/embj.2019102729 PMID: 31782550; PubMed Central PMCID: PMC6996502.

64. Brillen AL, Walotka L, Hillebrand F, Muller L, Widera M, Theiss S, et al. Analysis of Competing HIV-1 Splice Donor Sites Uncovers a Tight Cluster of Splicing Regulatory Elements within Exon $2 / 2 \mathrm{~b}$. J Virol. 2017; 91(14). Epub 2017/04/28. https://doi.org/10.1128/JVI.00389-17 PMID: 28446664; PubMed Central PMCID: PMC5487545.

65. Ludgate L, Liu K, Luckenbaugh L, Streck N, Eng S, Voitenleitner C, et al. Cell-Free Hepatitis B Virus Capsid Assembly Dependent on the Core Protein C-Terminal Domain and Regulated by Phosphorylation. J Virol. 2016; 90(12):5830-44. Epub 2016/04/15. https://doi.org/10.1128/JVI.00394-16 PMID: 27076641; PubMed Central PMCID: PMC4886785.

66. Zhao Q, Hu Z, Cheng J, Wu S, Luo Y, Chang J, et al. Hepatitis B Virus Core Protein Dephosphorylation Occurs during Pregenomic RNA Encapsidation. J Virol. 2018; 92(13). Epub 2018/04/20. https://doi org/10.1128/JVI.02139-17 PMID: 29669831; PubMed Central PMCID: PMC6002726.

67. Daub H, Blencke S, Habenberger P, Kurtenbach A, Dennenmoser J, Wissing J, et al. Identification of SRPK1 and SRPK2 as the major cellular protein kinases phosphorylating hepatitis B virus core protein. J Virol. 2002; 76(16):8124-37. PubMed https://doi.org/10.1128/jvi.76.16.8124-8137.2002 PMID: 12134018; PubMed Central PMCID: PMC155132.

68. Gazina EV, Fielding JE, Lin B, Anderson DA. Core protein phosphorylation modulates pregenomic RNA encapsidation to different extents in human and duck hepatitis B viruses. J Virol. 2000; 74 (10):4721-8. Epub 2000/04/25. https://doi.org/10.1128/jvi.74.10.4721-4728.2000 PMID: 10775610; PubMed Central PMCID: PMC111994.

69. Perlman DH, Berg EA, O'Connor P B, Costello CE, Hu J. Reverse transcription-associated dephosphorylation of hepadnavirus nucleocapsids. Proc Natl Acad Sci U S A. 2005; 102(25):9020-5. https:// doi.org/10.1073/pnas.0502138102 PMID: 15951426; PubMed Central PMCID: PMC1157036.

70. Ye F, Chen ER, Nilsen TW. Kaposi's Sarcoma-Associated Herpesvirus Utilizes and Manipulates RNA N(6)-Adenosine Methylation To Promote Lytic Replication. J Virol. 2017; 91(16). Epub 2017/06/09. https://doi.org/10.1128/JVI.00466-17 PMID: 28592530; PubMed Central PMCID: PMC5533915.

71. Mahiet C, Swanson CM. Control of HIV-1 gene expression by SR proteins. Biochem Soc Trans. 2016; 44(5):1417-25. Epub 2016/12/03. https://doi.org/10.1042/BST20160113 PMID: 27911724. 
72. Ji X, Zhou Y, Pandit S, Huang J, Li H, Lin CY, et al. SR proteins collaborate with 7SK and promoterassociated nascent RNA to release paused polymerase. Cell. 2013; 153(4):855-68. Epub 2013/05/ 15. https://doi.org/10.1016/j.cell.2013.04.028 PMID: 23663783; PubMed Central PMCID: PMC4103662.

73. Lin S, Coutinho-Mansfield G, Wang D, Pandit S, Fu XD. The splicing factor SC35 has an active role in transcriptional elongation. Nat Struct Mol Biol. 2008; 15(8):819-26. Epub 2008/07/22. https://doi.org/ 10.1038/nsmb.1461 PMID: 18641664; PubMed Central PMCID: PMC2574591.

74. Zhong XY, Wang P, Han J, Rosenfeld MG, Fu XD. SR proteins in vertical integration of gene expression from transcription to RNA processing to translation. Mol Cell. 2009; 35(1):1-10. Epub 2009/07/ 15. https://doi.org/10.1016/j.molcel.2009.06.016 PMID: 19595711; PubMed Central PMCID: PMC2744344.

75. Makokha GN, Abe-Chayama H, Chowdhury S, Hayes CN, Tsuge M, Yoshima T, et al. Regulation of the Hepatitis $B$ virus replication and gene expression by the multi-functional protein TARDBP. Sci Rep. 2019; 9(1):8462. Epub 2019/06/13. https://doi.org/10.1038/s41598-019-44934-5 PMID: 31186504; PubMed Central PMCID: PMC6560085.

76. Ng LF, Chan M, Chan SH, Cheng PC, Leung EH, Chen WN, et al. Host heterogeneous ribonucleoprotein K (hnRNP K) as a potential target to suppress hepatitis B virus replication. PLoS Med. 2005; 2(7): e163. Epub 2005/07/22. https://doi.org/10.1371/journal.pmed.0020163 PMID: 16033304; PubMed Central PMCID: PMC1181871.

77. Sun S, Nakashima K, Ito M, Li Y, Chida T, Takahashi H, et al. Involvement of PUF60 in Transcriptional and Post-transcriptional Regulation of Hepatitis B Virus Pregenomic RNA Expression. Sci Rep. 2017; 7(1):12874. Epub 2017/10/11. https://doi.org/10.1038/s41598-017-12497-y PMID: 28993636; PubMed Central PMCID: PMC5634508.

78. Tay N, Chan SH, Ren EC. Identification and cloning of a novel heterogeneous nuclear ribonucleoprotein C-like protein that functions as a transcriptional activator of the hepatitis B virus enhancer II. $\mathrm{J}$ Virol. 1992; 66(12):6841-8. Epub 1992/12/01. PubMed https://doi.org/10.1128/JVI.66.12.6841-6848. 1992 PMID: 1433497; PubMed Central PMCID: PMC240284.

79. Yao Y, Yang B, Cao H, Zhao K, Yuan Y, Chen Y, et al. RBM24 stabilizes hepatitis B virus pregenomic RNA but inhibits core protein translation by targeting the terminal redundancy sequence. Emerg Microbes Infect. 2018; 7(1):86. Epub 2018/05/16. https://doi.org/10.1038/s41426-018-0091-4 PMID: 29760415; PubMed Central PMCID: PMC5951808.

80. Imam H, Khan M, Gokhale NS, McIntyre ABR, Kim GW, Jang JY, et al. N6-methyladenosine modification of hepatitis B virus RNA differentially regulates the viral life cycle. Proc Natl Acad Sci U S A. 2018; 115(35):8829-34. Epub 2018/08/15. https://doi.org/10.1073/pnas.1808319115 PMID: 30104368; PubMed Central PMCID: PMC6126736.

81. Imam H, Kim GW, Mir SA, Khan M, Siddiqui A. Interferon-stimulated gene 20 (ISG20) selectively degrades N6-methyladenosine modified Hepatitis B Virus transcripts. PLoS Pathog. 2020; 16(2): e1008338. Epub 2020/02/15. https://doi.org/10.1371/journal.ppat.1008338 PMID: 32059034; PubMed Central PMCID: PMC7046284

82. Xiao W, Adhikari S, Dahal U, Chen YS, Hao YJ, Sun BF, et al. Nuclear m(6)A Reader YTHDC1 Regulates mRNA Splicing. Mol Cell. 2016; 61(4):507-19. Epub 2016/02/16. https://doi.org/10.1016/j. molcel.2016.01.012 PMID: 26876937.

83. Lucifora J, Arzberger S, Durantel D, Belloni L, Strubin M, Levrero M, et al. Hepatitis B virus X protein is essential to initiate and maintain virus replication after infection. J Hepatol. 2011; 55(5):996-1003. Epub 2011/03/08. https://doi.org/10.1016/j.jhep.2011.02.015 PMID: 21376091.

84. Lecluyse $\mathrm{EL}$, Alexandre $\mathrm{E}$. Isolation and culture of primary hepatocytes from resected human liver tissue. Methods Mol Biol. 2010; 640:57-82. Epub 2010/07/21. https://doi.org/10.1007/978-1-60761-6887_3 PMID: 20645046.

85. Ladner SK, Otto MJ, Barker CS, Zaifert K, Wang GH, Guo JT, et al. Inducible expression of human hepatitis $B$ virus (HBV) in stably transfected hepatoblastoma cells: a novel system for screening potential inhibitors of HBV replication. Antimicrob Agents Chemother. 1997; 41(8):1715-20. Epub 1997/08/ 01. PubMed https://doi.org/10.1128/AAC.41.8.1715 PMID: 9257747; PubMed Central PMCID: PMC163991.

86. Luangsay S, Gruffaz M, Isorce N, Testoni B, Michelet M, Faure-Dupuy S, et al. Early inhibition of hepatocyte innate responses by hepatitis B virus. J Hepatol. 2015; 63(6):1314-22. https://doi.org/10.1016/ j.jhep.2015.07.014 PMID: 26216533.

87. Hayer J, Jadeau F, Deleage G, Kay A, Zoulim F, Combet C. HBVdb: a knowledge database for Hepatitis B Virus. Nucleic Acids Res. 2013; 41(Database issue):D566-70. Epub 2012/11/06. https://doi.org/ 10.1093/nar/gks1022 PMID: 23125365; PubMed Central PMCID: PMC3531116. 
88. Dion S, Bourgine M, Godon O, Levillayer F, Michel ML. Adeno-associated virus-mediated gene transfer leads to persistent hepatitis B virus replication in mice expressing HLA-A2 and HLA-DR1 molecules. J Virol. 2013; 87(10):5554-63. https://doi.org/10.1128/JVI.03134-12 PMID: 23468504; PubMed Central PMCID: PMC3648192.

89. Salvetti A, Oreve S, Chadeuf G, Favre D, Cherel Y, Champion-Arnaud P, et al. Factors influencing recombinant adeno-associated virus production. Human Gene Therapy. 1998; 9(5):695-706. https:// doi.org/10.1089/hum.1998.9.5-695 WOS:000072839500010. PMID: 9551617

90. Mueller H, Wildum S, Luangsay S, Walther J, Lopez A, Tropberger P, et al. A novel orally available small molecule that inhibits hepatitis B virus expression. J Hepatol. 2018; 68(3):412-20. Epub 2017/ 10/29. https://doi.org/10.1016/j.jhep.2017.10.014 PMID: 29079285.

91. Ning X, Basagoudanavar SH, Liu K, Luckenbaugh L, Wei D, Wang C, et al. Capsid Phosphorylation State and Hepadnavirus Virion Secretion. J Virol. 2017; 91(9). https://doi.org/10.1128/JVI.00092-17 PMID: 28228589; PubMed Central PMCID: PMC5391479.

92. Salvetti A, Coute Y, Epstein A, Arata L, Kraut A, Navratil V, et al. Nuclear Functions of Nucleolin through Global Proteomics and Interactomic Approaches. J Proteome Res. 2016; 15(5):1659-69. https://doi.org/10.1021/acs.jproteome.6b00126 PMID: 27049334.

93. Tyanova S, Temu T, Cox J. The MaxQuant computational platform for mass spectrometry-based shotgun proteomics. Nat Protoc. 2016; 11(12):2301-19. Epub 2016/11/04. https://doi.org/10.1038/nprot. 2016.136 PMID: 27809316.

94. Schwanhausser B, Busse D, Li N, Dittmar G, Schuchhardt J, Wolf J, et al. Global quantification of mammalian gene expression control. Nature. 2011; 473(7347):337-42. Epub 2011/05/20. https://doi. org/10.1038/nature10098 PMID: 21593866.

95. Wieczorek S, Combes F, Lazar C, Giai Gianetto Q, Gatto L, Dorffer A, et al. DAPAR \& ProStaR: software to perform statistical analyses in quantitative discovery proteomics. Bioinformatics. 2017; 33 (1):135-6. https://doi.org/10.1093/bioinformatics/btw580 PMID: 27605098; PubMed Central PMCID: PMC5408771.

96. Bissig KD, Le TT, Woods NB, Verma IM. Repopulation of adult and neonatal mice with human hepatocytes: a chimeric animal model. Proc Natl Acad Sci U S A. 2007; 104(51):20507-11. Epub 2007/12/ 14. https://doi.org/10.1073/pnas.0710528105 PMID: 18077355; PubMed Central PMCID: PMC2154461.

97. Calattini S, Fusil F, Mancip J, Dao Thi VL, Granier C, Gadot N, et al. Functional and Biochemical Characterization of Hepatitis C Virus (HCV) Particles Produced in a Humanized Liver Mouse Model. The Journal of biological chemistry. 2015; 290(38):23173-87. Epub 2015/08/01. https://doi.org/10.1074/ jbc.M115.662999 PMID: 26224633; PubMed Central PMCID: PMC4645586.

98. de Chaumont F, Dallongeville S, Chenouard N, Herve N, Pop S, Provoost T, et al. Icy: an open bioimage informatics platform for extended reproducible research. Nat Methods. 2012; 9(7):690-6. Epub 2012/06/30. https://doi.org/10.1038/nmeth.2075 PMID: 22743774.

99. Lucifora J, Xia Y, Reisinger F, Zhang K, Stadler D, Cheng X, et al. Specific and nonhepatotoxic degradation of nuclear hepatitis B virus cccDNA. Science. 2014; 343(6176):1221-8. https://doi.org/10.1126/ science.1243462 PMID: 24557838.

100. Werle-Lapostolle B, Bowden S, Locarnini S, Wursthorn K, Petersen J, Lau G, et al. Persistence of cccDNA during the natural history of chronic hepatitis $B$ and decline during adefovir dipivoxil therapy. Gastroenterology. 2004; 126(7):1750-8. Epub 2004/06/10. https://doi.org/10.1053/j.gastro.2004.03. 018 PMID: 15188170

101. Alfaiate D, Lucifora J, Abeywickrama-Samarakoon N, Michelet M, Testoni B, Cortay JC, et al. HDV RNA replication is associated with HBV repression and interferon-stimulated genes induction in superinfected hepatocytes. Antiviral Res. 2016; 136:19-31. https://doi.org/10.1016/j.antiviral.2016.10.006 PMID: 27771387.

102. Brosseau JP, Lucier JF, Lapointe E, Durand M, Gendron D, Gervais-Bird J, et al. High-throughput quantification of splicing isoforms. RNA. 2010; 16(2):442-9. Epub 2009/12/30. https://doi.org/10. 1261/rna.1877010 PMID: 20038630; PubMed Central PMCID: PMC2811672.

103. Prinos P, Garneau D, Lucier JF, Gendron D, Couture S, Boivin M, et al. Alternative splicing of SYK regulates mitosis and cell survival. Nat Struct Mol Biol. 2011; 18(6):673-9. Epub 2011/05/10. https://doi. org/10.1038/nsmb.2040 PMID: 21552259.

104. Smits AH, Jansen PW, Poser I, Hyman AA, Vermeulen M. Stoichiometry of chromatin-associated protein complexes revealed by label-free quantitative mass spectrometry-based proteomics. Nucleic Acids Res. 2013; 41(1):e28. Epub 2012/10/16. https://doi.org/10.1093/nar/gks941 PMID: 23066101; PubMed Central PMCID: PMC3592467. 